\title{
Measuring the tolerance of the genetic code to altered codon size
}

\author{
E. DeBenedictis ${ }^{1,2 *}$, D. Söll ${ }^{3}$, K. Esvelt ${ }^{2}$ \\ ${ }^{1}$ Department of Biological Engineering, Massachusetts Institute of Technology. \\ ${ }^{2}$ Department of Media Arts and Sciences, Massachusetts Institute of Technology. \\ ${ }^{3}$ Department of Molecular Biophysics and Biochemistry, Yale University. \\ Correspondence: erika.alden@mit.edu
}

\section{Summary}

Protein translation using four-base codons occurs in both natural and synthetic systems. What constraints contributed to the universal adoption of a triplet-codon, rather than quadruplet-codon, genetic code? Here, we investigate the tolerance of the $E$. coli genetic code to tRNA mutations that increase codon size. We found that tRNAs from all twenty canonical isoacceptor classes can be converted to functional quadruplet tRNAs (qtRNAs), many of which selectively incorporate a single amino acid in response to a specified four-base codon. However, efficient quadruplet codon translation often requires multiple tRNA mutations, potentially constraining evolution. Moreover, while tRNAs were largely amenable to quadruplet conversion, only nine of the twenty aminoacyl tRNA synthetases tolerate quadruplet anticodons. These constitute a functional and mutually orthogonal set, but one that sharply limits the chemical alphabet available to a nascent all-quadruplet code. Our results illuminate factors that led to selection and maintenance of triplet codons in primordial Earth and provide a blueprint for synthetic biologists to deliberately engineer an all-quadruplet expanded genetic code.

\section{INTRODUCTION}

The genetic code is determined by a combination of tRNAs and aminoacyl tRNA synthetases (AARSs). Codons are dictated by the three bases in the center of the anticodon loop of each tRNA, which undergo Watson-Crick base pairing to an mRNA transcript during translation, enabling accurate codon recognition. The correspondence between codons and amino acids - one tRNA isoacceptor class for each canonical amino acid - is dictated by the twenty AARSs which specifically recognize bases (identity elements) in the tRNAs, and attach the cognate amino acid onto only the CCA 3' terminus of the cognate tRNA. The aminoacylation process is exquisitely accurate, enabling highfidelity protein synthesis ${ }^{1}$. Anticodon mutations frequently alter or abolish selective charging with the cognate amino acid because most AARSs rely on bases in the anticodon to identify the cognate tRNA ${ }^{2}$. However, certain natural anticodon mutations generate 'suppressor' tRNAs that insert their cognate amino acid in response to 5'-UAG-3' stop codons using a cognate 5'-CUA-3' anticodon ${ }^{3}$. Frameshift suppression, in which a quadruplet tRNA reads through a frameshift mutation, are also known to arise naturally ${ }^{4,5}$; i.e. qtRNA-Gly-GGGG can decode the four-base codon 5'-GGGG-3' in mRNA transcripts using the 5'-CCCC-3' anticodon. In the presence of efficiently aminoacylated qtRNAs, the ribosome is capable of protein translation with individual non-canonical stop or quadruplet codons within an otherwise all-triplet transcript ${ }^{6}$. If individual quadruplet codon translation is known to arise through simple point insertions, what functional constraints, if any, prevent the natural or synthetic evolution of an all-quadruplet genetic code?

These origin-of-life questions have newfound importance to engineering with the advent of genetic code expansion technology. An expanded all- quadruplet genetic code would offer 256 total codons, including hundreds of free codons that could be assigned to noncanonical amino acids (ncAAs), valuable chemical additions to the genetic code that enable improved protein therapeutics ${ }^{7}$. Most work to date has focused on incorporating ncAAs using quadruplet codons within otherwise all-triplet mRNA transcripts ${ }^{8}$. An all-quadruplet genetic code would presumably require the ability to incorporate the 20 canonical amino acids with quadruplet codons. Might the necessary translation components be easily derived from existing tRNAs and AARSs?

To investigate these questions, we assessed the tolerance of the genetic code to mutations that alter codon size in E. coli. We systematically explored whether quadruplet codon translation can arise through simple point insertions in each of the tRNA anticodon loops, or through mutation of many bases in the anticodon, then used directed evolution to determine how often additional mutations throughout the tRNA can improve translation of the resulting quadruplet qtRNAs. Finally, we characterized the fidelity quadruplet codon translation. Remarkably, we found that $12 / 20$ isoacceptor classes of tRNAs can be readily converted to selectively charged qtRNAs, that activity is low but can often be improved by accumulating additional mutations along the sides of the anticodon loop, and that most of the resulting qtRNAs selectively incorporate a single amino acid in response to a quadruplet codon. Our results suggest that quadruplet codon translation was undoubtedly explored by primordial organisms, identifies the barriers limiting its complete adoption by natural evolution, and presents 9/20 qtRNAs necessary to create an all-quadruplet expanded genetic code.

\section{Evolution of quadruplet tRNAs (qtRNAs)}
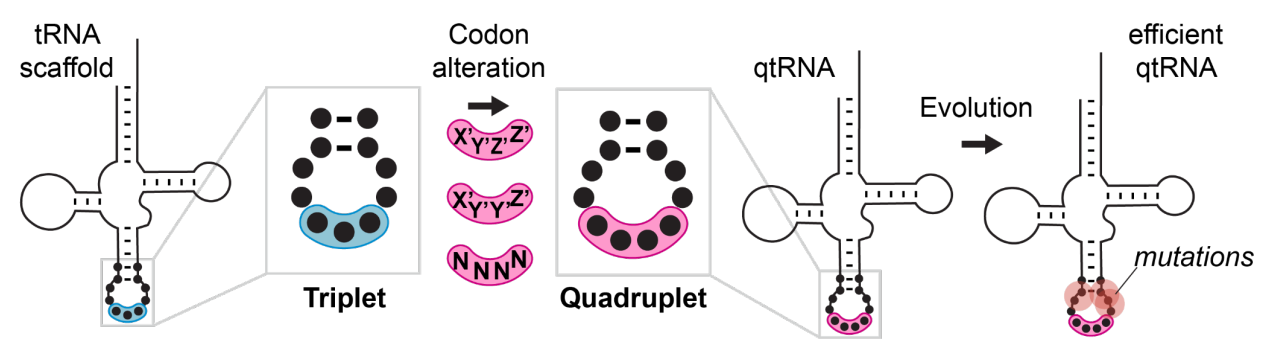

Visual abstract: We studied whether tRNAs can arise through simple changes to the anticodon followed by additional mutations accumulated during evolution. 
bioRxiv preprint doi: https://doi.org/10.1101/2021.04.26.441066; this version posted April 26, 2021. The copyright holder for this preprint (which was not certified by peer review) is the author/funder, who has granted bioRxiv a license to display the preprint in perpetuity. It is made available under aCC-BY-NC-ND 4.0 International license.

\section{RESULTS}

\section{Evolution of qtRNAs through point insertions.}

Many known examples of frameshift suppressors contain single point insertions in the anticodon that convert a triplet tRNA into a quadruplet tRNA (qtRNA). We initially tested whether tRNAs can evolve into qtRNAs through simple point insertions. We selected 21 endogenous $E$. coli tRNAs, one cognate tRNA for each canonical amino acid and initiator methionine (Table S1), to serve as 'scaffolds' into which we introduce anticodon point insertions. We tested two point insertion locations: if the original tRNA decodes the triplet codon " $X Y Z$ ", we created a qtRNA that decodes "XYZZ" and a qtRNA that decodes "XYYZ" (Figure 1A). These anticodon patterns preserve the nature of the bases in the anticodon loop, which most AARSs use to recognize the cognate tRNA ${ }^{2}$, and are found in known qtRNAs, such as sufD ${ }^{4}$ (qtRNA ${ }^{\text {Gly }}{ }_{G G G G}$ ) and sufG ${ }^{9}$ (qtRNA ${ }^{\text {Gln }}$ CAAA). Throughout, for ease of comparison to standard triplet codon tables, we use qtRNA ${ }^{\text {three letter scaffold }}$ four letter DNA codon nomenclature to refer to qtRNAs; e.g. a serine qtRNA bearing a 5'-UCUA-3' anticodon is referred to as qtRNA ${ }^{\text {Ser }}$ TAGA.

We used two techniques to characterize these qtRNAs. First, to measure the quadruplet codon translation efficiency, we used a luciferase readthrough assay. This reporter contains a single quadruplet codon at permissive residue 357 of luxAB; failure to decode the quadruplet codon leads to premature termination, whereas successful four base decoding results in full-length luxAB translation and luminescence (Figure 1B).
Seven of the twenty "XYZZ"-decoding qtRNAs and two of the "XYYZ"decoding qtRNAs functionally decode a quadruplet codon during translation. The frequent functionality of the $X Y Z Z$ codon pattern may be due to flexibility in synthetase recognition at the third position of the codon, which is frequently a wobble base pair. Next, we quantified the toxicity of qtRNA expression by comparing the growth of cultures with qtRNA expression induced or suppressed (Figure 1C). The qtRNAs fall into several categories: those that exhibit no fitness defect such as the naturally occurring and highly functional qtRNA ${ }^{\text {Gly }}$ GGGG; those that exhibit severe fitness defects and effectively halt bacterial growth upon induction such as qtRNA ${ }^{\mathrm{Ala}}{ }_{\mathrm{GCCC}}$, and those that moderately slow bacterial growth. The translation efficiency and growth defect of qtRNAs depends upon more than just the interaction with the cognate AARS: AlaRS, LeuRS, and SerRS are all synthetases that do not interact with the anticodon loop of their cognate tRNA, but qtRNAs derived from these scaffolds exhibit a range of behaviors depending upon the new anticodon. For example, qtRNA ${ }^{\text {Ser }}{ }_{\text {TCCG }}$ exhibits high growth defect, while qtRNA ${ }^{\text {Ser }}$ TCGG exhibits no growth defect and modest quadruplet translation efficiency. Together, this data shows that a third of the twenty isoacceptor classes have access to point insertions that create modestly functional quadruplet codon translation, however, other point insertions create qtRNAs that do not functionally decode quadruplet codons or incur large growth defects when expressed.
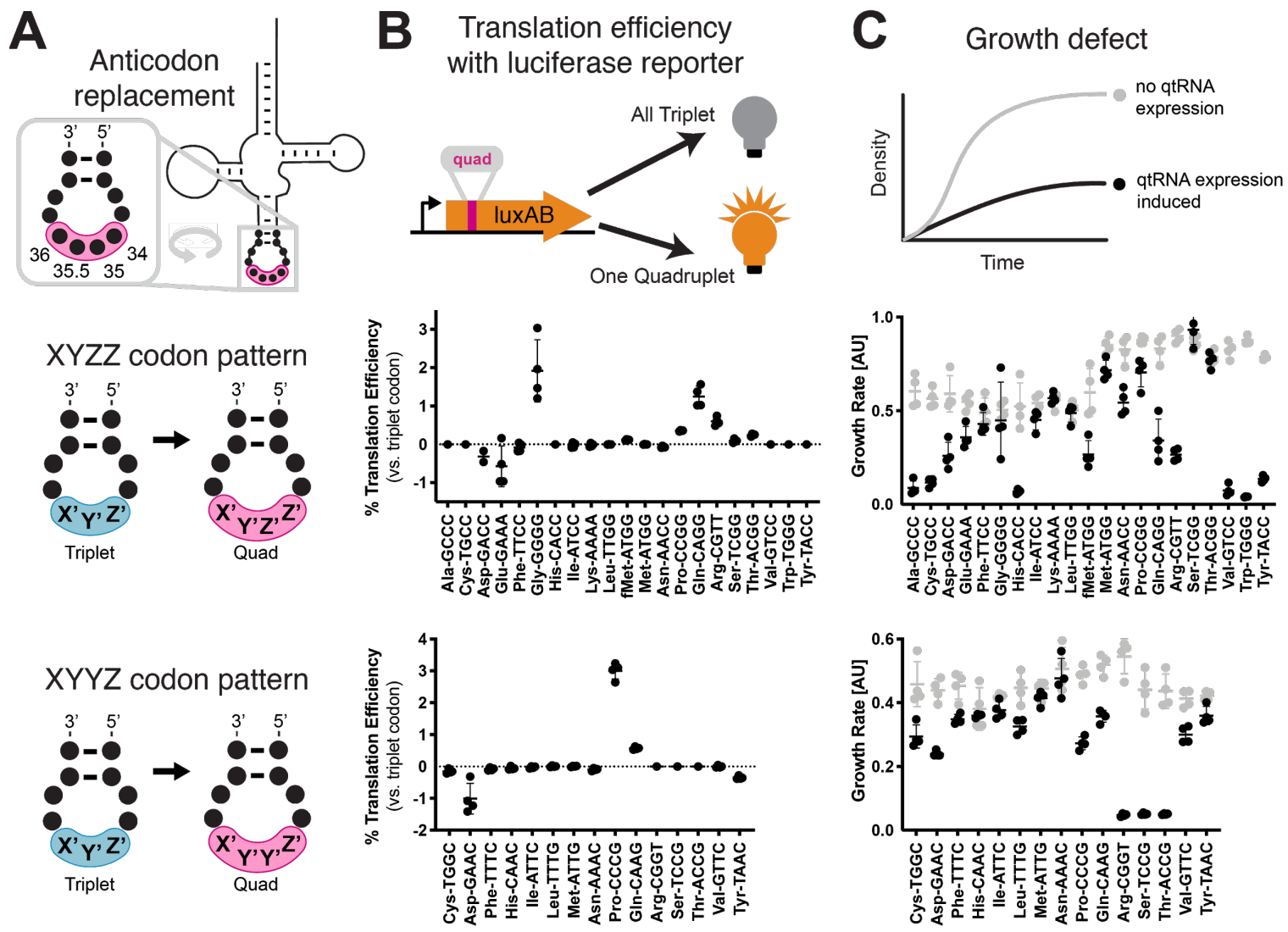

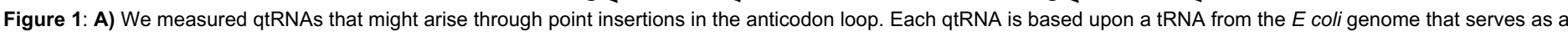

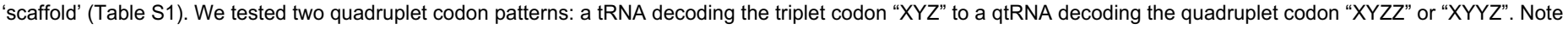

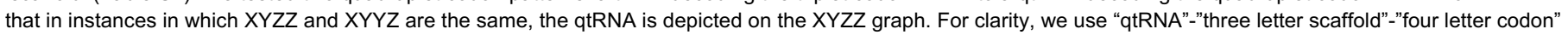

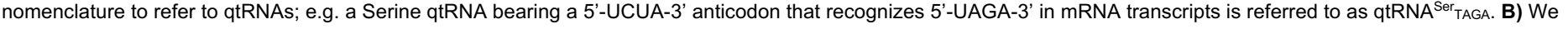
measured these qtRNAs using a luciferase readthrough assay. In the presence of a functional qtRNA generates full-length luxAB transcript and luminescence, while nonfunctional qtRNAs generate a truncated luxAB transcript and no luminescence. Measurements are taken kinetically and normalized to culture density. Efficiency is

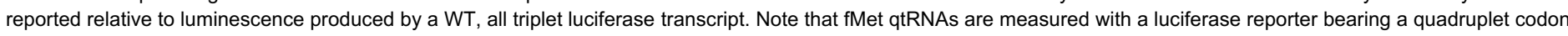

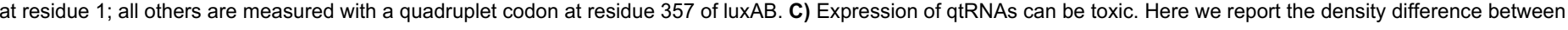
cultures where qtRNA expression had been induced (black) or suppressed (grey). 
bioRxiv preprint doi: https://doi.org/10.1101/2021.04.26.441066; this version posted April 26, 2021. The copyright holder for this preprint (which was not certified by peer review) is the author/funder, who has granted bioRxiv a license to display the preprint in perpetuity. It is made available under aCC-BY-NC-ND 4.0 International license.

\section{Evolution of qtRNAs through anticodon replacement.}

Next, we tested whether tRNAs could evolve into qtRNAs through whole anticodon replacement, as might occur during recombination or more intense mutagenesis. Antibiotic selection markers have previously been used to identify functional qtRNAs ${ }^{10}$. We applied an equivalent approach based on the use of $\mathrm{M} 13$ bacteriophage tail fiber plll as a selection marker ${ }^{11}$. For each of the 20 representative E. coli tRNA scaffolds used above, we created a 256-member qtRNA library containing degenerate anticodons (Figure $2 \mathrm{~A}$ ) and selected for functional qtRNAs from these libraries (Figure 2B) to identify qtRNAs that decode any of eight quadruplet codons of interest (Figure $2 \mathrm{C}$ ). High final phage titers after selection indicate the presence of a functional qtRNA, so we used phage titer to guide us in selecting 69 putative qtRNAs for further characterization (Figure 2D). Using Sanger sequencing, we determined the anticodon of each highly selected variant. We found that for most variants, the qtRNA agrees with the quadruplet codon in the reporter at all four positions, or the first three positions of the codon (Figure 2E), in agreement with previous findings on quadruplet codon crosstalk with fourth-base mismatches ${ }^{12}$. We found that qtRNAs identified by this phage-based assay are also functional in the luciferase assay. These results demonstrate that most tRNA scaffolds are capable of supporting quadruplet codon translation through whole-anticodon replacement.

In addition to the expected anticodons, we found that some qtRNAs instead matched quadruplet codons that appear nearby in the sequence context of the reporter (Figure 2F). We noticed that the unexpected codons often bear similarity to the qtRNA's original codon; i.e. qtRNA ${ }^{\text {Lys }}$ AGAA is highly similar to the scaffold's original AAA codon, and qtRNA ${ }^{\text {Tyr }}$ TACA and qtRNA ${ }^{\text {Phe }}$ TACA $_{\text {are }}$ similar to TAC and TTC respectively. The emergence of these anticodons suggests that these isoacceptor classes favor quadruplet codons that are related to their natural triplet codon, and demonstrates that qtRNA evolution depends upon the sequence context of relevant ORFs.

Together, these experiments identified functional qtRNAs involving four or fewer mutations for $18 / 20$ isoacceptor classes. For the remaining two isoacceptor classes, Met and Asn, we systematically tested additional codons and found that qtRNA ${ }^{\text {Met }}{ }_{\text {AGGG }}$ and qtRNA ${ }^{A s n}{ }_{A G G A}$ both exhibit weak quadruplet codon translation (Figure S1). Therefore, every isoacceptor class can give rise to qtRNAs capable of decoding quadruplet codons during protein translation.

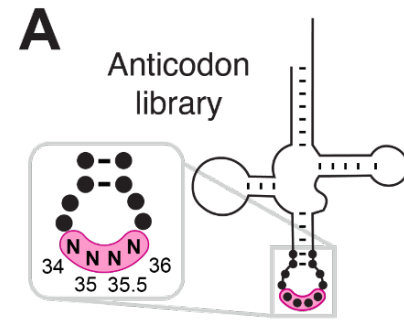

qtRNA on phage

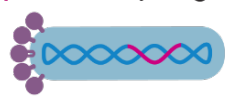

Pre-selection

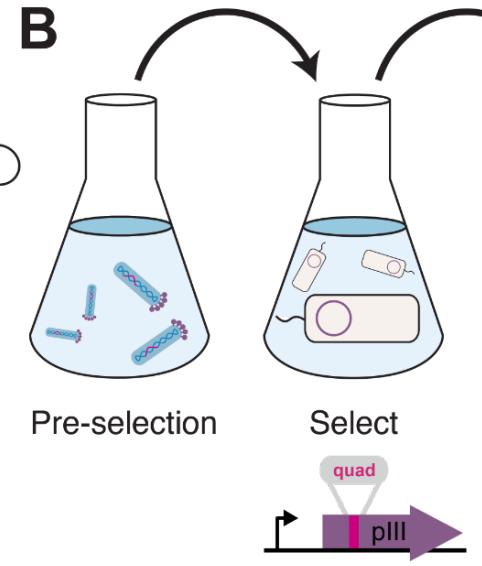

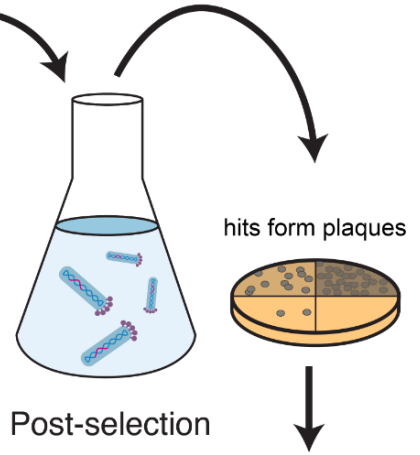

Sanger sequence

C

20 Anticodon Libraries

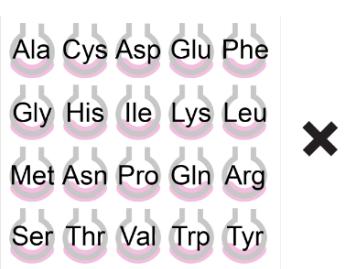

$\rightarrow 160$ Separate Library Selections 8 Selection Conditions AGGG CGGT CGTT GGGG
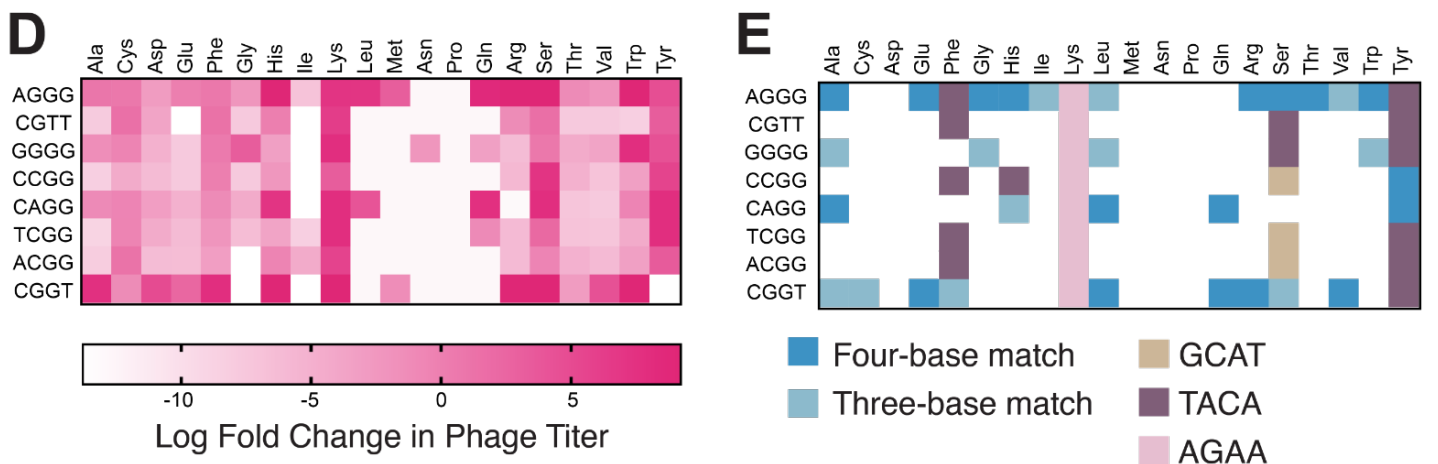

$E$

plll residues 28 - 33 aaaACGGcatacalgaaat $\begin{array}{llllll}\mathrm{K} & \sum_{28} & \mathrm{H} & \mathrm{T} & \mathrm{E} & \mathrm{N} \\ 30 & 31 & 32 & 33\end{array}$

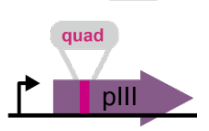

Log Change in Phage Titer

Figure 2: A) We created qtRNA libraries using degenerate primers to randomize the four bases in the anticodon. The qtRNA library is expressed from a $\triangle \mathrm{plII} M 13$

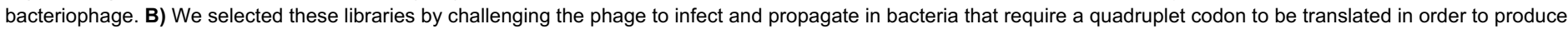

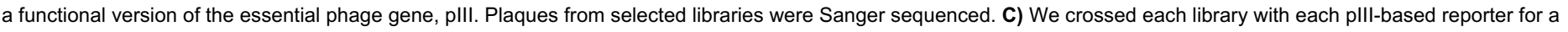

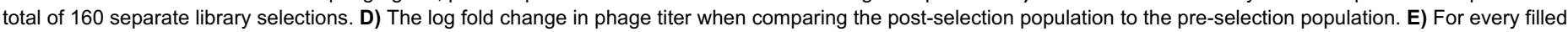

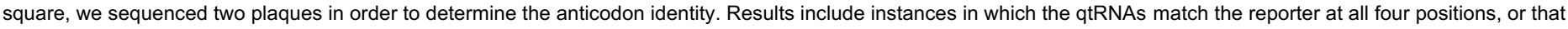

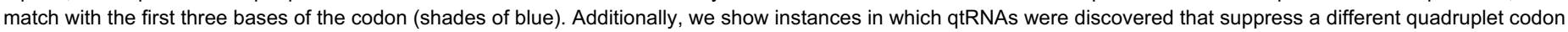

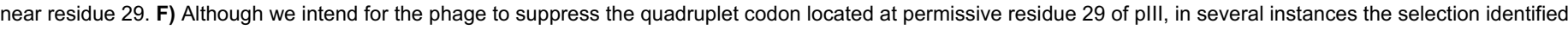
qtRNAs that suppress a nearby quadruplet codon instead. 
bioRxiv preprint doi: https://doi.org/10.1101/2021.04.26.441066; this version posted April 26, 2021. The copyright holder for this preprint (which was not certified by peer review) is the author/funder, who has granted bioRxiv a license to display the preprint in perpetuity. It is made available under aCC-BY-NC-ND 4.0 International license.

\section{Directed evolution of qtRNAs}

Having found that qtRNAs that functionally decode quadruplet codons can arise generally through just a few mutations, we sought to understand other factors that may prevent more widespread use of quadruplet codons. Many qtRNAs were quite inefficient in translation: the presence of a single quadruplet codon in an mRNA transcript can reduce total protein yield to less than $3 \%$ relative to an all-triplet mRNA. Mutations at the anticodon loop sides of the qtRNAs have been observed to improve translation efficiency for TAGA-qtRNAs ${ }^{11,13,36}$. Triplet tRNAs are known to exhibit patterns that relate the bases in the ALS to the bases in the anticodon itself ${ }^{14}$, and similarly benefit from ALS mutations after anticodon replacement ${ }^{15-17}$. In some cases, mutations in this area can alter qtRNA charging; in others they improve quadruplet translation efficiency without altering the qtRNA's interaction with the cognate AARS $^{36}$. We hypothesized that qtRNAs in general require mutations at bases 32, 37 and 38 to better accommodate a new codon, and that this requirement may present a key barrier preventing the natural evolution of efficient qtRNAs.

To test this hypothesis experimentally, we selected 41 promising qtRNAs, including at least one qtRNA for every unique scaffold, and cloned a library containing degenerate nucleotides at bases 32,37 , and 38 (Figure $3 \mathrm{~A})$. We selected functional members of these libraries using the plllbased selection and used next generation sequencing to characterize the abundance of each library member before and after selection (Figure 3B).

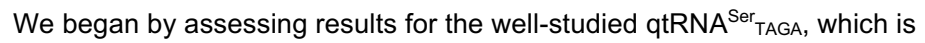
known to have an improved variant, qtRNA ${ }^{\text {Ser }}{ }_{\text {TAGA }}-32 A-38 \mathrm{C}$, that exhibits improved quadruplet codon translation but unaltered, selective aminoacylation with serine ${ }^{36}$ (Figure $3 C$ ). Of the 64 possible combinations of DNA bases at positions 32,37 , and 38 , the single library member A32 A37 C38 is enriched four log fold above all other variants (Figure 3D). We measured several qtRNA ${ }^{\text {Ser }}$ TAGA variants that correspond to different levels of enrichment using a luciferase readthrough assay, and confirmed that the strongly enriched variant exhibits more efficient quadruplet decoding than deenriched variants (Figure 3E).

Next, we applied the same procedure to quantify fold enrichment for the other 40 qtRNA libraries (Figure 3F). We identified 15 libraries that exhibit no selective pressure, 12 libraries that strongly enrich a single library

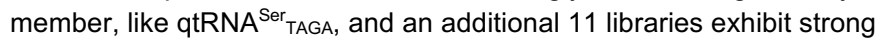
enrichment for library members with a specific base at one or two positions, but not all three (Figure 3G). For several of these libraries, we used a luciferase readthrough assay to measure the fold change activity when mutations to bases 32, 37 and 38 are introduced. In most cases, introduction of these mutations substantially increases quadruplet codon translation efficiency (Figure $3 \mathrm{H}$ ).

We were curious whether there are overall trends in the identity of optimal anticodon loop sides for efficient quadruplet codon translation. The optimal library member is not determined by the scaffold or codon independently, indicating that the mechanism by which these mutations improve quadruplet codon translation does not improve the qtRNA's interaction with its respective AARS. Amongst the libraries there was a prominent preference for $A 37$, a base known to be associated with reading frame maintenance ${ }^{18}$. Additionally, C32 A37 T38 and T32 A37 G38 appear often amongst libraries that exhibit strong preference for a single library member. The presence of modified nucleotides is especially important at two sites: position 34, the first base of the anticodon, and position 37 , the nucleotide downstream of the anticodon ${ }^{18,20}$. The location of 'identity elements' for some of these modifying enzymes within the anticodon loop sides has been implied ${ }^{20}$. Mutation of these bases may improve the RNA modification of the anticodon loop, which are essential for many tRNA functions ${ }^{19}$. 
bioRxiv preprint doi: https://doi.org/10.1101/2021.04.26.441066; this version posted April 26, 2021. The copyright holder for this preprint (which was not certified by peer review) is the author/funder, who has granted bioRxiv a license to display the preprint in perpetuity. It is made available under aCC-BY-NC-ND 4.0 International license.

A

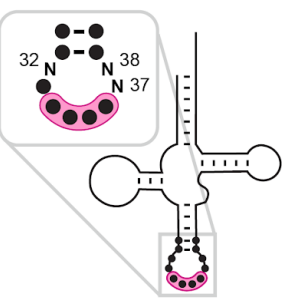

qtRNA on phage

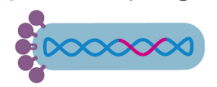

B

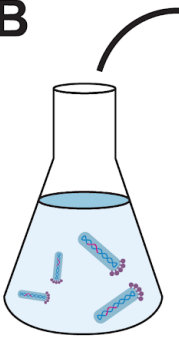

Pre-selection

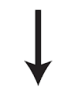

NGS

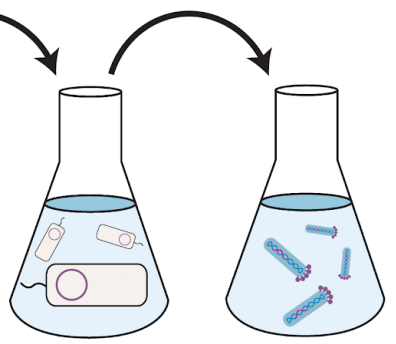

Select

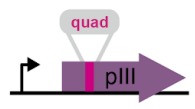

Post-selection

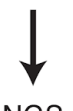

NGS

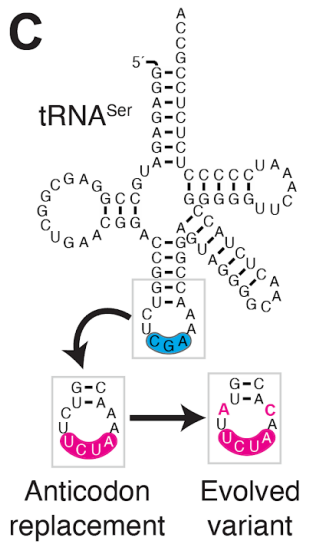

replacement variant

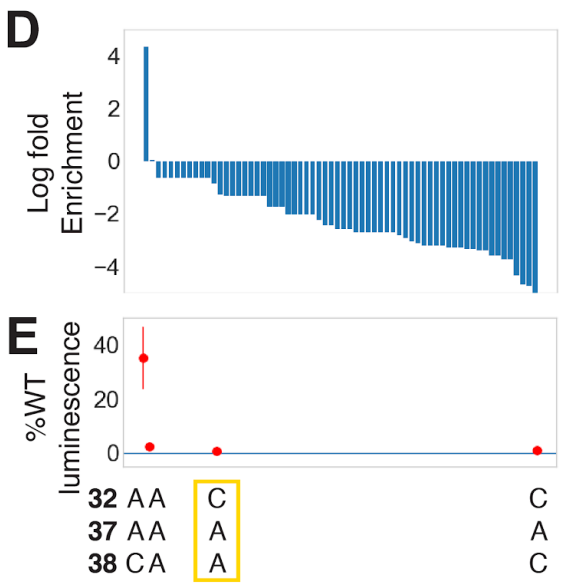

F

Log fold enrichment

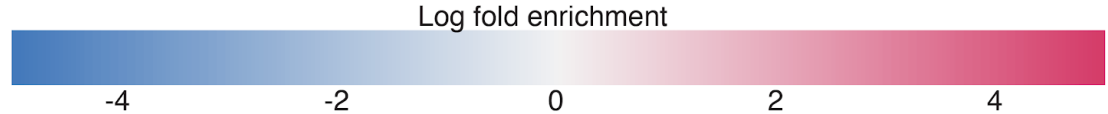

WT

G $\quad$ H

Trend Fold Activity

323738 Change

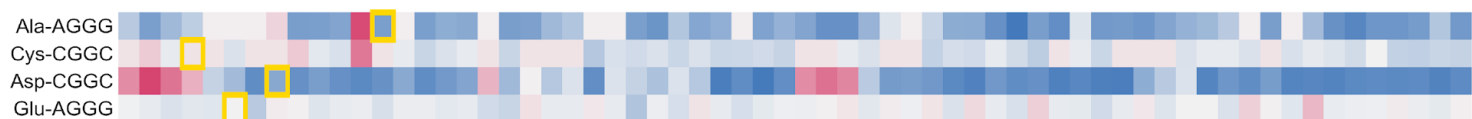

Glu-AGGG

Glu-CGGC

Phe-CGGC

Phe-TACA

Gly-AGGG

Gly-GGGG

Gly-TAGA

His-AGGG

His-TACA

Lys-AGAA

Pro-CCGG

GIn-CAGG

Arg-CGGC

Ser-AGGG

Ser-CGGC

Ser-TACA

Ser-TAGA

Ser-TCGG

Thr-AGGG

Val-CGGC

Trp-GGGT

Tyr-TACA

Glu-TAGA
His-TAGA

His-TAGA
Cys-TAGA

Asp-AGGG

Ile-AGGG

Leu-AGGG

Met-AGGG

Asn-AGGG

Arg-AGGG

Arg-CGTT

Thr-ACGG

Trp-AGGG

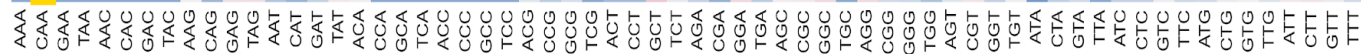

base 32 - base 37 - base 38

Figure 3: A) We created qtRNA libraries using degenerate primers to randomize positions 32,37 and 38 of the anticodon. The qtRNA library is expressed from a $\triangle$ plII $M 13$ bacteriophage. B) We selected these libraries by challenging the phage to infect and propagate in bacteria that require a quadruplet codon to be translated in order to produce

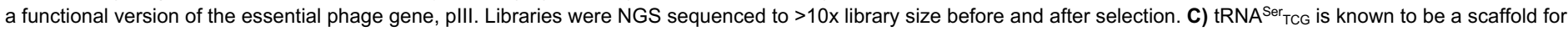

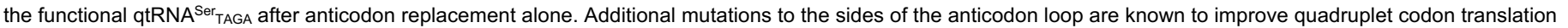
efficiency. D) Log fold enrichment of the population abundance and E) translation efficiency as measured by a luciferase readthrough assay of the 64 possible combinations of nucleotides at positions 32, 37 and 38. F) Log fold enrichment of all 41 qtRNA libraries for each of the 64 library members. Libraries are separated by those that exhibit abundance changes during selection (above) from those that exhibit no significant abundance changes (below). G) For each library, the trend in nucleotide preference for each position is listed. $\mathbf{H}$ ) Nucleotide preferences for select libraries were measured by cloning a qtRNA variant and measuring it using a luciferase readthrough assay. The fold improvement in activity over the wildtype values of base 32,37 and 38 are listed. In e) and f), the original identities of bases 32,37 , and 38 found in the wildtype triplet tRNA scaffold are boxed in gold. 
bioRxiv preprint doi: https://doi.org/10.1101/2021.04.26.441066; this version posted April 26, 2021. The copyright holder for this preprint (which was not certified by peer review) is the author/funder, who has granted bioRxiv a license to display the preprint in perpetuity. It is made available under aCC-BY-NC-ND 4.0 International license.

\section{Which amino acid(s) do qtRNAs incorporate during translation?}

Having established that anticodon modification can often produce tRNAs that decode four-base codons, we sought to determine which amino acid these qtRNAs incorporate during translation. To do so, we translated sfGFP mRNA containing a quadruplet codon at permissive residue 151 in the presence of a qtRNA. We then used mass spectral analysis to determine the nature and occupancy of amino acid 151 in the resulting protein ${ }^{17}$ for qtRNAs based on the 20 distinct tRNA scaffolds (Figure 4).

We were unable to characterize the acylation properties of six qtRNAs due to low sfGFP purification yield, even when purified at $1-L$ scale (Table S2) (qtRNA ${ }^{\text {Asn }}$, qtRNA ${ }^{\text {Cys }}$, qtRNA ${ }^{\text {Leu }}$, qtRNA ${ }^{\text {Lys }}$, qtRNA ${ }^{\text {Met }}$, and qtRNA ${ }^{\text {Thr }}$ ). These qtRNAs would be unlikely to be selected in a natural system due to their inability to produce full-length protein efficiently. Eight qtRNAs are selectively acylated with the amino acid cognate to the original scaffold (qtRNA ${ }^{\text {Arg }}$, qtRNA $^{\text {Gln }}$, qtRNA $A^{\text {Glu }}$, qtRNA ${ }^{\text {Gly }}$, qtRNA ${ }^{\text {Phe }}$, qtRNA ${ }^{\text {Pro, }}$, $q^{\text {qtRNA }}{ }^{\text {Ser }}$, qtRNA ${ }^{\text {Tyr }}$ ). Four qtRNAs selectively incorporate Arg, rather than the amino acid cognate to the scaffold (qtRNA ${ }^{\text {Asp }}$, qtRNA ${ }^{\text {lle }}$, qtRNA ${ }^{\text {Trp }}$, qtRNA ${ }^{\text {Val }}$ ). Two qtRNAs that are charged with the cognate amino acid as well as promiscuously charged with Arg (qtRNA ${ }^{\text {Ala }}$ and qtRNA ${ }^{\text {Met }}$ ). All 14 of these qtRNAs might be selected by a natural system to rescue a frameshift mutation. The 12 qtRNAs that are selectively charged might be further selected as components of an all-quadruplet genetic code.

These data highlight the plasticity of AARS recognition for altered codons, both in nucleobase composition and size. The presence of a quadruplet anticodon presumably distorts the structure of the anticodon binding domain, which is a major identity element for many AARSs ${ }^{2}$. How much distortion will the enzyme functionally accept, and/or will recognition of the additional base lead to mischarging with a different amino acid? Of the eight qtRNAs that retained the cognate specificity, tRNA ${ }^{\text {Gly }}$ and tRNAPro are known as naturally occurring functional frameshift suppressors ${ }^{4,21}$; we confirm that they are selectively charged by the cognate AARS. Together with tRNA ${ }^{\mathrm{Gin}}$, our results show that $E$. coli GInRS, GlyRS and ProRS recognize the first three bases of the quadruplet anticodon, and are capable of charging qtRNAs despite the increased anticodon size. Correct charging of qtRNAs derived from qtRNA ${ }^{\text {Ser }}$ is expected, as E. coli SerRS does not interact with the anticodon ${ }^{22}$. A striking result is the correct charging of qtRNA ${ }^{\text {Glu }}{ }_{\text {CGGT }}$ and qtRNA ${ }^{\text {Phe }}{ }_{\text {CGGC }}$ by their respective $E$. coli tRNA synthetases, as their triplet anticodon sequence is unlike that of the quadruplet anticodon. However, in both cases all other known critical identity elements ${ }^{2}$ are present in the tRNA scaffold. This means that the major recognition feature, the anticodon, can be outweighed by the sum of the other identity elements, creating in some cases an avenue for anticodon evolution. qTRNA-Glu-CGGT

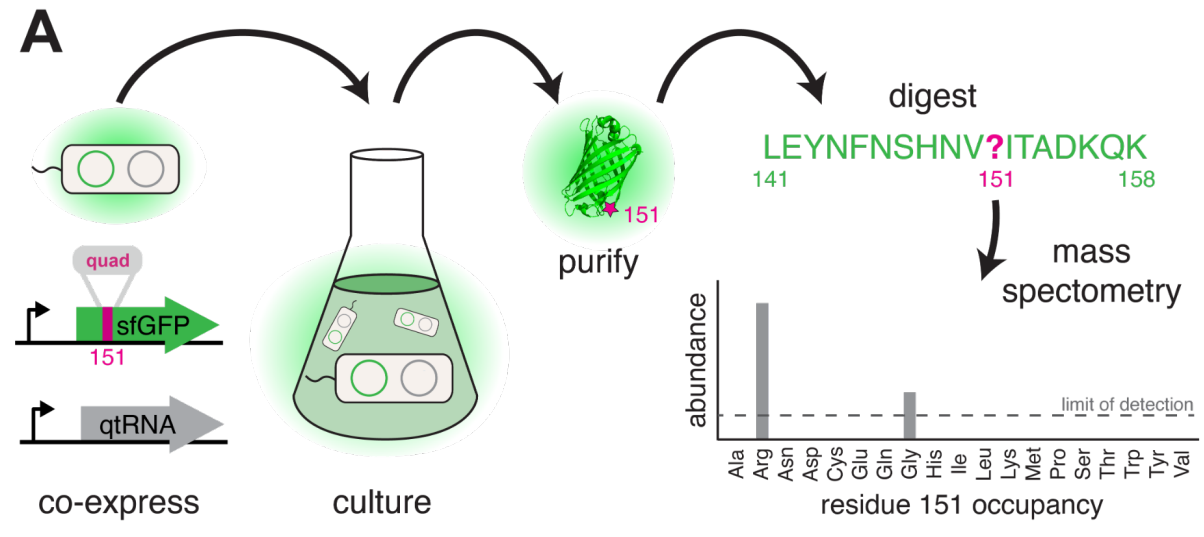

\section{B qtRNA Amino Acid Incorporation}

qtRNA-Ala-AGGG-32T-38G qtRNA-Asn-AGGA qtRNA-Asp-CGGC-32G-38A qtRNA-Cys-CGGC 促 qtRNA-His-TACA-32 qtRNA-lle-AGGA qRNA-Leu-AGGG qRA-LYS-AGAA qtRNA-Phe-CGGC qtRNA-Pro-CCGG qtRNA-Thr-ACGG qtRNA-Trp-AGGG qtRNA-Tyr-TAGA

Figure 4: Characterization of amino acid incorporation by qtRNAs. A) We characterized the amino acid incorporated during translation by co-expressing a qtRNA and a sfGFP-151-quad transcript, purifying the resulting GFP, and analyzing the occupancy of residue 151 using mass spectrometry. B) Results of applying too low to allow for charging characterization even when purified at $1 \mathrm{~L}$ scale (Table S2). Charging of $\left({ }^{*}\right)$ qtRNA ${ }^{\text {Arg }}{ }_{\text {TAGA }}$ and qtRNA ${ }^{\text {Tyr }}$ TAGA were first reported in [36]

$77 \%$ Arg; $22 \%$ Ala
$100 \%$ Arg $^{*}$
-
$100 \%$ Arg
-
$100 \%$ Glu
$100 \%$ Gln
$100 \%$ Gly
-
$100 \%$ Arg
-
-
$96 \%$ Arg; $3 \%$ Met; $2 \%$ Gly
$100 \%$ Phe
$100 \%$ Pro
$100 \%$ Ser
-
$100 \%$ Arg
$100 \%$ Tyr
$100 \%$ Arg

Low yield- cannot purify Promiscuous Selective - same amino acid Selective - altered amino acid
Finally, why did we observe acylation of multiple qtRNAs with arginine? ArgRS is responsible for synthesis of a family of Arg-tRNAs needed for the recognition of six codons, including tRNAs that differ at both the first and last position of the anticodon. As a consequence, the identity of just one anticodon position is invariant (C35). Examination of the qtRNAs charged with Arg (Table 4B) shows that all satisfy this anticodon identity element, causing rampant promiscuous charging with Arg, even for qtRNAs that lack other known argRS identity elements such as A20 such

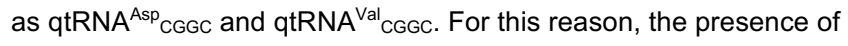
promiscuous ArgRS substantially increases the probability that tRNA point insertions in diverse scaffolds will result in an aminoacylated qtRNA.

Taken together, we found that in the majority of cases, qtRNAs exhibit properties that would render them evolutionarily favored building blocks: they are selectively charged by a single amino acid and efficiently incorporate that amino acid in response to their quadruplet codon. 
bioRxiv preprint doi: https://doi.org/10.1101/2021.04.26.441066; this version posted April 26, 2021. The copyright holder for this preprint (which was not certified by peer review) is the author/funder, who has granted bioRxiv a license to display the preprint in perpetuity. It is made available under aCC-BY-NC-ND 4.0 International license.

A

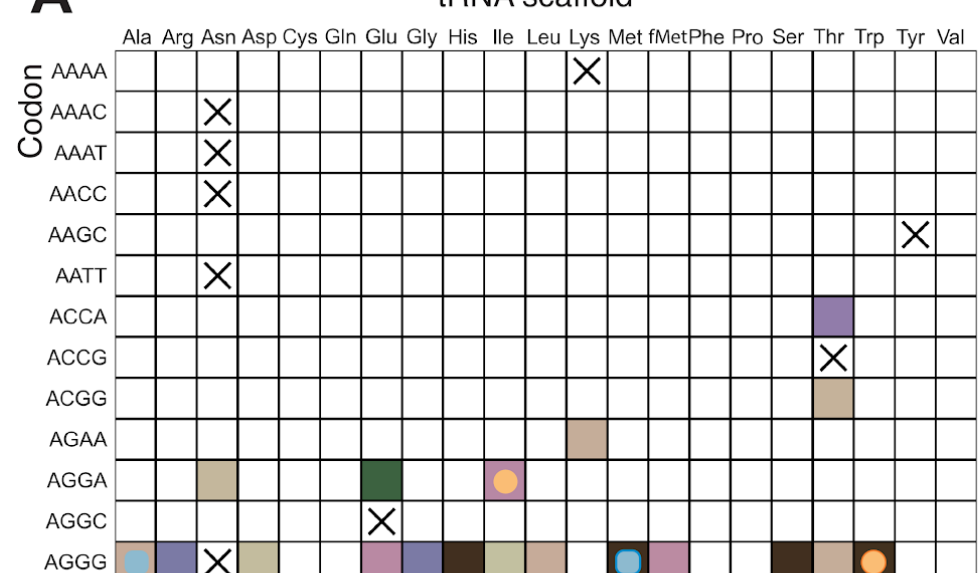

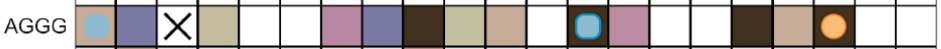

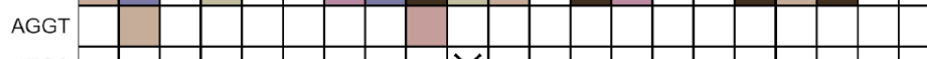

ATCC

ATGG

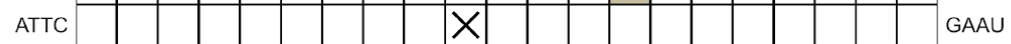

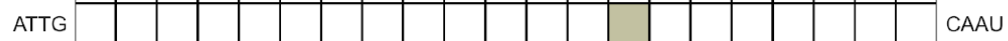

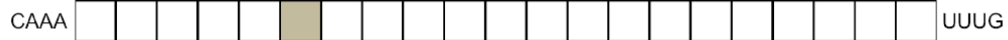

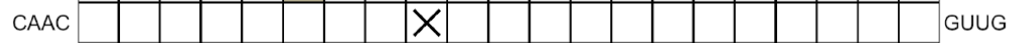

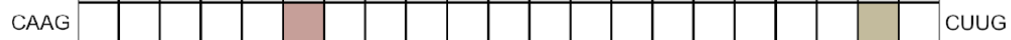

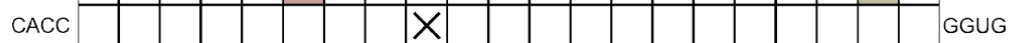

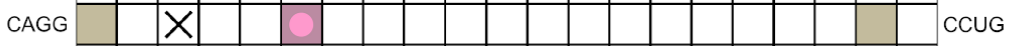

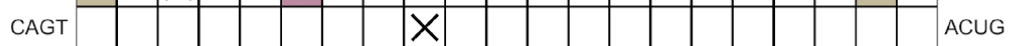

CATG

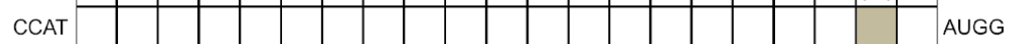

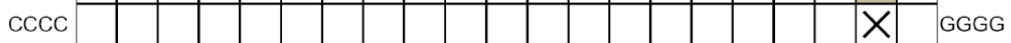

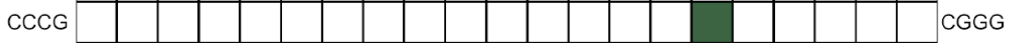

ССCT

CCGG

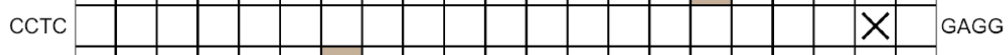

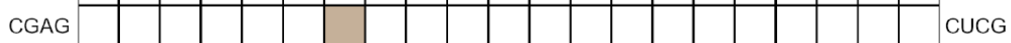

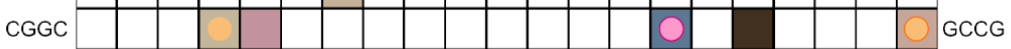

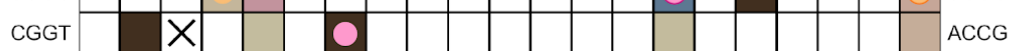

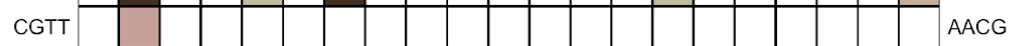

GAAA

GAAC

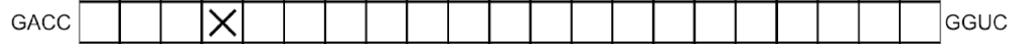

GCAT

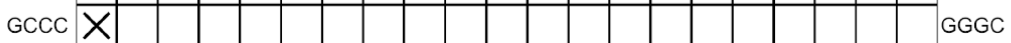

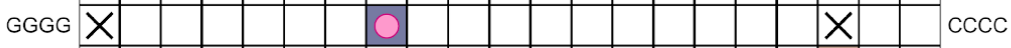

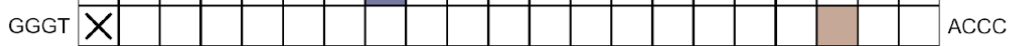

GTCC

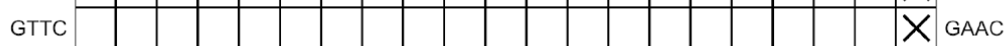

TAAC

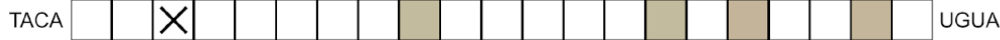

TACC

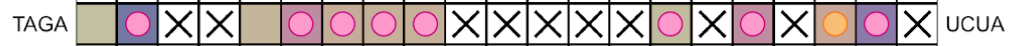

TCCG

TCGG

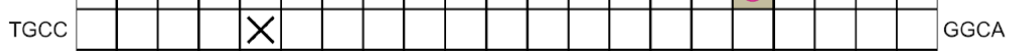

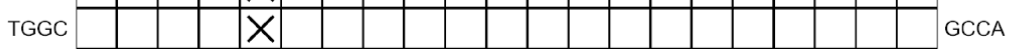

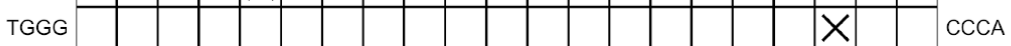

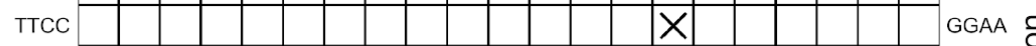

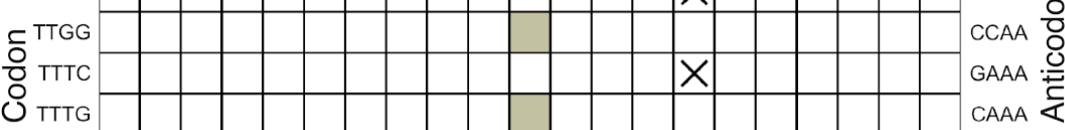

Ala Arg Asn Asp Cys Gln Glu Gly His lle Leu Lys MetfMet Phe Pro Ser Thr Trp Tyr Val

tRNA scaffold

\section{Translation Efficiency}

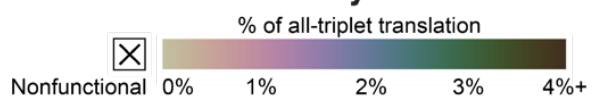

\section{Amino Acid Incorporation}

Promiscuous

Selective - same amino acid

Selective - altered amino acid

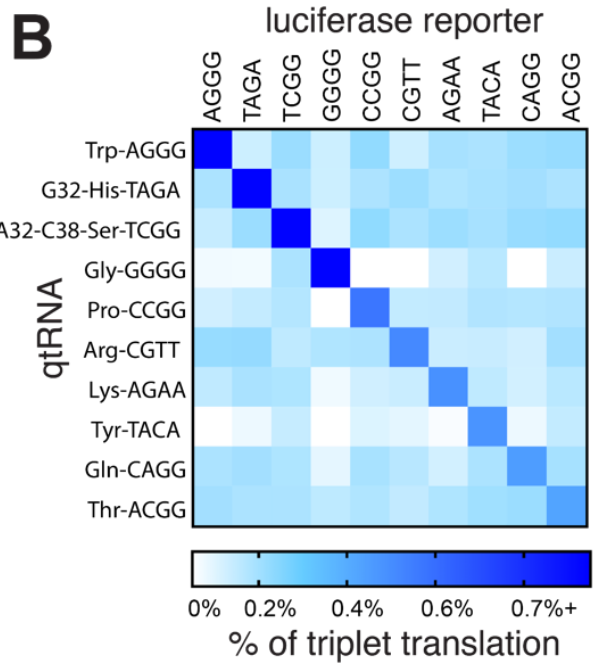

Figure 5: A) Compiled results of quantifying qtRNA translation efficiency (luciferase redthrough assay) and charging (GFP mass spectrometry). The qtRNA scaffold (columns) and codon (rows) are indicated. The translation efficiency is indicated on each qtRNA that could be measured, or marked as "nonfunctional", meaning the qtRNA exhibits too strong a growth defect to measure, or does not produce a measurable increase luminescence upon induction of qtRNA expression. Translation efficiency is measured as a percent of all-triplet translation (see Methods). Note that fMet qtRNAs are measured with a luciferase reporter bearing a quadruplet codon at residue 1 ; all others are measured with a quadruplet codon at residue 357 of luxAB. Charging results are indicated on the table for qtRNAs derived from each scaffoldcodon pair; measured qtRNAs may contain additional mutations; see Table S2. B) A miniature all-quadruplet genetic code. For each of the 10 qtRNAs (rows), we measured readthrough of a luciferase transcript containing the indicated quadruplet codon at residue 357 (columns). 


\section{Trends in nascent qtRNA evolution.}

In total, we characterized 116 different qtRNAs based on 20 tRNA scaffolds that decode 20 unique quadruplet codons (Figure $5 \mathrm{~A}$ ). This vastly expands the total number of known qtRNAs, the diversity of triplet tRNA scaffolds they are based upon, and the diversity of quadruplet codons they recognize beyond what has previously been reported. We found that 60 out of 109 are functional, that is, they generate increased luminescence upon induction of qtRNA expression. In total, every tRNA scaffold we tested is capable of supporting quadruplet codon translation given appropriate four-base codon choice.

Could these codon-diverse qtRNAs, if accumulated in a genome, enable all-quadruple translation? To be used as part of a genetic code, qtRNAs must be capable of operating together with minimal crosstalk. We selected 10 qtRNAs and measured crosstalk between members of this set, revealing a high degree of orthogonality: each qtRNA is specific for its own four-base codon (Figure 5B). Additionally, we found eight novel qtRNAs that selectively incorporate the same amino acid as the scaffold, despite the altered codon. Together, nine different AARSs (ArgRS, GluRS, GInRS, GlyRS, HisRS, PheRS, ProRS, SerRS, TyrRS) faithfully and efficiently charge a cognate qtRNA, creating the opportunity for amino acid and codon-diverse all quadruplet peptide translation.

\section{DISCUSSION}

Primordial Earth certainly sampled qtRNAs during early evolution: we found that every tRNA in E. coli is no more than 4 mutations away from supporting quadruplet codon translation. Indeed, single point insertions in the anticodon generate functional qtRNAs for $7 / 20$ isoacceptor classes. The most efficient of these, such as qtRNA ${ }^{\text {Gly }}{ }_{G G G G}$, have been discovered as naturally occurring frameshift suppressors ${ }^{4,5}$, indicating that existing approaches have been successful in identifying only the most efficient qtRNAs. There are an additional 5 isoacceptor classes that can support reasonably efficient quadruplet codon translation with whole-anticodon replacement. We found that $12 / 20$ isoacceptor classes result in qtRNAs that are selectively charged by a single amino acid, making them putative components of novel, high-fidelity genetic codes. Additionally, the diversity of codons that can be recognized by qtRNAs can easily enable codon-selective translation, as we demonstrate with a miniature allquadruplet genetic code composed of 10 orthogonal qtRNAs based on unique isoacceptor classes. Together, these results show that qtRNAs certainly arose many times during evolution in primordial Earth, and that many of the qtRNAs were capable of amino-acid selective, efficient translation of quadruplet codons.

However, our study identifies several factors that may have prevented universal adoption of an all-quadruplet code. First, we find that some qtRNAs exhibit strong fitness defects which prevent them from being maintained and accumulated in genomes. Second, additional mutations outside the anticodon are often required for efficient translation, reducing the probability that efficient qtRNAs would arise by chance. Such mutations may affect identity elements of the tRNA modifying enzymes that chemically alter the bases surrounding the anticodon ${ }^{23}$; similar patterns have been observed for canonical triplet tRNAs ${ }^{14}$. Finally, only some AARSs are amenable to altered codon sizes for some amino acids: 12/20 modern E. coli AARSs are intolerant, inefficient, or promiscuous with qtRNAs. In particular, AARSs that arose later during evolution may be more finely tuned to precise anticodon base recognition and thus less tolerant of anticodon mutations or expansion. A nascent all-quadruplet code would initially have access to only the limited chemical vocabulary of the nine AARSs that are most amenable to quadruplet codon translation, decreasing the likelihood that valuable quadruplet-ORFs might arise and be selected. Finally, studies have shown that ORFs can also arise de novo from non-coding regions ${ }^{24}$ and that random ORFs can rapidly evolve into functional proteins ${ }^{25}$, offering an avenue for gene birth in a different codon size should the appropriate qtRNAs be available. However, even in an organism that had accumulated several functional qtRNAs, meaningful de novo quadruplet ORFs are less likely to arise by chance than equivalent triplet ORFs due to the expanded number of possible codons ( 256 vs. 64 ) and the reduced initial number of sense codons ( 8 vs. 61$)$. Together, these factors contribute to the absence of naturally occurring quadruplet codon codes, despite the frequency with which individual quadruplet translation components can arise through evolution.

Although these factors explain the absence of naturally occurring allquadruplet genetic codes, our results offer a clear blueprint for deliberately engineering an expanded all-quadruplet genetic code. With 256 total codons, this genetic code would create hundreds of free codons that could be assigned to noncanonical amino acids (ncAAs), valuable chemical additions to the genetic code that enable improved protein therapeutics ${ }^{7}$. Our data has revealed, for the first time, exactly which triplet codons can be reassigned to quadruplet codons without compromising amino acid selectivity: an initial all-quadruplet code will be composed of 9 selectively charged qtRNAs from this study (qtRNA ${ }^{\mathrm{Gln}}{ }_{\mathrm{CAGG}}$, qtRNA ${ }^{\text {Pro }}{ }_{C C G G}, q t R N A^{\text {Phe }}{ }_{C G G C}, q t R N A^{G l u}{ }_{C G G T}$, qtRNA ${ }^{\text {Gly }}{ }_{G G G G}, q t R N A^{\text {Ser }_{T C G G}}$, qtRNA ${ }^{\mathrm{Arg}}{ }_{\mathrm{AGGG}}$, and either qtRNA ${ }^{\mathrm{Tyr}_{\mathrm{TAGA}}}$ or qtRNA ${ }^{\mathrm{His}}{ }_{\text {TAGA }}$ ). Further, the flexibility of AARSs suggests that anticodon reengineering may be a viable strategy for reassignment of the additional 11 canonical amino acids to quadruplet codons. We and others ${ }^{26}$ have observed that ArgRS is a major source of promiscuous charging; refactoring or replacing this ArgRS may be broadly valuable for enabling reassignment of argininelike codons; i.e. enabling selective charging of qtRNA ${ }^{\mathrm{Ala}}{ }_{\mathrm{AGGG}}$. Additionally, strategies such as CCA tail engineering ${ }^{27,28}$ will be required to mitigate toxicity and competition with triplet tRNAs. Together, our deliberate exploration of the evolution of quadruplet translation has provided components that will launch synthetic efforts to assemble a 256-amino acid genetic code.

\section{Acknowledgements}

We thank Eric Spooner and the Whitehead Proteomics Core Facility. We thank the laboratory of Kristala Prather for equipment use and assistance. We thank Nili Ostrov, Ben Thuroni, Stephen Von Stetina, and Sergey Melnikov for their helpful comments on the manuscript. We thank BiologicsCorp for protein purification.

\section{Funding}

This work was supported by the MIT Media Lab, an Alfred P. Sloan Research Fellowship (to K.M.E.), gifts from the Open Philanthropy Project and the Reid Hoffman Foundation (to K.M.E.), and the National Institute of Digestive and Kidney Diseases (R00 DK102669-01 to K.M.E.), and by the National Institute of General Medical Sciences (R35GM122560 to D.S.). E.A.D. was supported by the National Institute for Allergy and Infectious Diseases (F31 Al145181-01).

\section{Author contributions}

E.A.D conceived the study. E.A.D designed the experiments with advice from K.M.E. E.A.D performed the experiments. K.M.E supervised the research. E.A.D wrote the manuscript with input from all authors.

\section{Competing interests}

E.A.D and K.M.E have filed a patent application with the US Patent and Trademark Office on this work.

\section{Data and materials availability}

Key plasmids from this study have been deposited on Addgene. 
bioRxiv preprint doi: https://doi.org/10.1101/2021.04.26.441066; this version posted April 26, 2021. The copyright holder for this preprint (which was not certified by peer review) is the author/funder, who has granted bioRxiv a license to display the preprint in perpetuity. It is made available under aCC-BY-NC-ND 4.0 International license.

\section{References}

1. Reynolds, N. M., Lazazzera, B. A. \& Ibba, M. Cellular mechanisms that control mistranslation. Nat. Rev. Microbiol. 8, 849-856 (2010).

2. Giegé, R., Sissler, M. \& Florentz, C. Universal rules and idiosyncratic features in tRNA identity. Nucleic Acids Res. 26, 5017-5035 (1998).

3. Eggertsson, G. \& Söll, D. Transfer ribonucleic acid-mediated suppression of termination codons in Escherichia coli. Microbiol. Rev. 52, 354-374 (1988).

4. Riddle, D. L. \& Carbon, J. Frameshift suppression: a nucleotide addition in the anticodon of a glycine transfer RNA. Nat. New Biol. 242, 230-234 (1973).

5. Roth, J. R. Frameshift suppression. Cell 24, 601-602 (1981).

6. Dunkelmann, D. L., Willis, J. C. W., Beattie, A. T. \& Chin, J. W. Engineered triply orthogonal pyrrolysyl-tRNA synthetase/tRNA pairs enable the genetic encoding of three distinct non-canonical amino acids. Nat. Chem. 12, 535-544 (2020).

7. Hutchins, B. M. et al. Site-specific coupling and sterically controlled formation of multimeric antibody fab fragments with unnatural amino acids. $J$. Mol. Biol. 406, 595-603 (2011).

8. de la Torre, D. \& Chin, J. W. Reprogramming the genetic code. Nat. Rev. Genet. 22, 169-184 (2021).

9. O'Connor, M. Insertions in the anticodon loop of tRNA1GIn(sufG) and tRNALys promote quadruplet decoding of CAAA. Nucleic Acids Res. 30, 1985-1990 (2002).

10. Magliery, T. J., Anderson, J. C. \& Schultz, P. G. Expanding the genetic code: selection of efficient suppressors of four-base codons and identification of 'shifty' four-base codons with a library approach in Escherichia coli. Edited by M. Gottesman. J. Mol. Biol. 307, 755-769 (2001).

11. DeBenedictis, E. A., Chory, E. J., Gretton, D., Wang, B. \& Esvelt, K. A high-throughput platform for feedback-controlled directed evolution. bioRxiv 2020.04.01.021022 (2020) doi:10.1101/2020.04.01.021022.

12. Anderson, J. C., Magliery, T. J. \& Schultz, P. G. Exploring the limits of codon and anticodon size. Chem. Biol. 9, 237-244 (2002).

13. Niu, W., Schultz, P. G. \& Guo, J. An expanded genetic code in mammalian cells with a functional quadruplet codon. ACS Chem. Biol. 8, 1640-1645 (2013).

14. Yarus, M. Translational efficiency of transfer RNA's: uses of an extended anticodon. Science 218, 646-652 (1982).

15. Kleina, L. G., Masson, J. M., Normanly, J., Abelson, J. \& Miller, J. H. Construction of Escherichia coli amber suppressor tRNA genes. II. Synthesis of additional tRNA genes and improvement of suppressor efficiency. J. Mol. Biol. 213, 705-717 (1990).

16. Raftery, L. A. \& Yarus, M. Systematic alterations in the anticodon arm make tRNA (Glu)-Suoc a more efficient suppressor. EMBO J. 6, $1499-1506$ (1987).

17. Cervettini, D. et al. Rapid discovery and evolution of orthogonal aminoacyl-tRNA synthetase-tRNA pairs. Nat. Biotechnol. (2020) doi:10.1038/s41587-020-0479-2.

18. Agris, P. F. Decoding the genome: a modified view. Nucleic Acids Res. 32, 223-238 (2004).

19. Edwards, A. M., Addo, M. A. \& Dos Santos, P. C. Extracurricular Functions of tRNA Modifications in Microorganisms. Genes 11, (2020).

20. Grosjean, H. \& Westhof, E. An integrated, structure- and energy-based view of the genetic code. Nucleic Acids Res. 44, 8020-8040 (2016).

21. Sroga, G. E., Nemoto, F., Kuchino, Y. \& Björk, G. Insertion ( sufB ) in the anticodon loop or base substitution ( sufC ) in the anticodon stem of tRNA Pro2 from Salmonella typhimurium induces suppression of frameshift mutations. Nucleic Acids Res. 20, 3463-3469 (1992).

22. Biou, V., Yaremchuk, A., Tukalo, M. \& Cusack, S. The 2.9 A crystal structure of T. thermophilus seryl-tRNA synthetase complexed with tRNA(Ser). Science 263, 1204-1410 (1994).

23. Hori, H. Methylated nucleosides in tRNA and tRNA methyltransferases. Front. Genet. 5, 144 (2014).

24. Carvunis, A.-R. et al. Proto-genes and de novo gene birth. Nature 487, 370-374 (2012).

25. Keefe, A. D. \& Szostak, J. W. Functional proteins from a random-sequence library. Nature 410, 715-718 (2001).

26. Krishnakumar, R. et al. Transfer RNA misidentification scrambles sense codon recoding. Chembiochem 14, 1967-1972 (2013).

27. Samaha, R. R., Green, R. \& Noller, H. F. A base pair between tRNA and $23 \mathrm{~S}$ rRNA in the peptidyl transferase centre of the ribosome. Nature 377 , 309-314 (1995).

28. Terasaka, N., Hayashi, G., Katoh, T. \& Suga, H. An orthogonal ribosome-tRNA pair via engineering of the peptidyl transferase center. Nat. Chem. Biol. 10, 555-557 (2014).

29. Bryson, D. I. et al. Continuous directed evolution of aminoacyl-tRNA synthetases. Nat. Chem. Biol. (2017) doi:10.1038/nchembio.2474.

30. Geu-Flores, F., Nour-Eldin, H. H., Nielsen, M. T. \& Halkier, B. A. USER fusion: a rapid and efficient method for simultaneous fusion and cloning of multiple PCR products. Nucleic Acids Research vol. 35 e55-e55 (2007).

31. Hubbard, B. P. et al. Continuous directed evolution of DNA-binding proteins to improve TALEN specificity. Nat. Methods 12, 939-942 (2015).

32. Badran, A. H. et al. Continuous evolution of Bacillus thuringiensis toxins overcomes insect resistance. Nature 533, 58-63 (2016).

33. Weinberg, Z. \& Breaker, R. R. R2R--software to speed the depiction of aesthetic consensus RNA secondary structures. BMC Bioinformatics 12, 3 (2011).

34. Chory, E. J., Gretton, D. W., Debenedictis, E. A. \& Esvelt, K. M. Flexible open-source automation for robotic bioengineering. bioRxiv (2020).

35. Young, D. D. et al. An evolved aminoacyl-tRNA synthetase with atypical polysubstrate specificity. Biochemistry 50, 1894-1900 (2011).

36. DeBenedictis, E. A., Chung, C., Soll, D. \& Badran, A. H. Continuous Directed Evolution of tRNAs Improves Quadruplet Codon Translation. (in review) 
bioRxiv preprint doi: https://doi.org/10.1101/2021.04.26.441066; this version posted April 26, 2021. The copyright holder for this preprint (which was not certified by peer review) is the author/funder, who has granted bioRxiv a license to display the preprint in perpetuity. It is made available under aCC-BY-NC-ND 4.0 International license.

\section{Methods}

\section{General methods}

Antibiotics. Antibiotics (Gold Biotechnology) were used at the following working concentrations: carbenicillin, $50 \mu \mathrm{g} / \mathrm{mL}$; spectinomycin, 100 $\mu \mathrm{g} / \mathrm{mL}$; chloramphenicol, $40 \mu \mathrm{g} / \mathrm{mL}$; kanamycin, $30 \mu \mathrm{g} / \mathrm{mL}$; tetracycline, $10 \mu \mathrm{g} / \mathrm{mL}$; streptomycin, $50 \mu \mathrm{g} / \mathrm{mL}$.

Media. David Rich Medium (DRM) ${ }^{29}$ (US biological, \#CS050H-001 and \#CS050H-003) is used for luminescence assays due to its low fluorescence and luminescence background. 2XYT media (US biological, T9200), a media optimized for phage growth, is used for all other purposes, including phage-based selection assays and general cloning. Agar (US biological, A0930) is used for cloning and plaque assays.

Plasmid cloning. tRNA genes were amplified directly from E. coli genomic DNA; see Table S1 for tRNA sequences and links to Benchling plasmid maps. Plasmids were cloned using either Mach1, Turbo, DH5a, or 10beta cells. Unless otherwise noted, plasmids and phage were cloned by USER assembly ${ }^{30}$ using the Phusion U Hot Start DNA polymerase (Thermofisher, F556L) and USER enzyme (New England Biolabs, M5505L).

Preparation of chemically competent cells. Strain S2060 (Addgene \#105064), a K12 derivative optimized for directed evolution ${ }^{31}$ was used in all luciferase, phage propagation, and plaque assays. To prepare competent cells, an overnight culture was diluted 1,000 -fold into $50 \mathrm{~mL}$ of 2XYT media supplemented with maintenance antibiotics and grown at 37 ${ }^{\circ} \mathrm{C}$ with shaking at $230 \mathrm{rpm}$ to $\mathrm{OD}_{600} \sim 0.4-0.6$. Cells were pelleted by centrifugation at $6,000 \times \mathrm{g}$ for $10 \mathrm{~min}$ at $4{ }^{\circ} \mathrm{C}$. The cell pellet was then resuspended by gentle stirring in $5 \mathrm{~mL}$ of TSS (LB media supplemented with $5 \%$ v/v DMSO, 10\% w/v PEG 3350, and $20 \mathrm{mM} \mathrm{MgCl}_{2}$ ). The cell suspension was stirred to mix completely, aliquoted and flash-frozen in liquid nitrogen, and stored at $-80^{\circ} \mathrm{C}$ until use.

Transformation of chemically competent cells. To transform cells, $100 \mu \mathrm{L}$ of competent cells were thawed on ice. To this, plasmid $(2 \mu \mathrm{L}$ each of miniprep-quality plasmid; up to two plasmids per transformation) and 100 $\mu \mathrm{L} \mathrm{KCM}$ solution ( $100 \mathrm{mM} \mathrm{KCl}, 30 \mathrm{mM} \mathrm{CaCl}$, and $50 \mathrm{mM} \mathrm{MgCl}_{2}$ in $\mathrm{H}_{2} \mathrm{O}$ ) were added and stirred gently with a pipette tip. The mixture was incubated on ice for $10 \mathrm{~min}$ and heat shocked at $42{ }^{\circ} \mathrm{C}$ for $45 \mathrm{~s}$. The mixture was chilled on ice for $4 \mathrm{~min}$, then $850 \mu \mathrm{L}$ of 2XYT media was added. Cells were allowed to recover at $37^{\circ} \mathrm{C}$ with shaking at $230 \mathrm{rpm}$ for $0.75 \mathrm{~h}$, streaked on $2 \mathrm{XYT}$ media $+1.5 \%$ agar plates containing the appropriate antibiotics, and incubated at $37^{\circ} \mathrm{C}$ for $16-18 \mathrm{~h}$.

Standard phage cloning. Competent E. coli S2060 cells were prepared containing pJC175e (Addgene \#79219), a plasmid expressing plll under control of the phage shock promoter ${ }^{32}$, which enables propagation of $\Delta$ plII M13 bacteriophage through complementation. To clone $\Delta$ pIII M13 bacteriophage, PCR fragments were assembled using USER. The annealed fragments were transformed into competent S2060-pJC175e competent cells. Transformants were recovered in 2XYT media overnight, shaking at $230 \mathrm{rpm}$ at $37^{\circ} \mathrm{C}$. The phage supernatant from the resulting culture was filtered through a $0.22 \mu \mathrm{m}$ membrane (Thomas Scientific, 1166U41), and plaqued to isolate clonal phage (see below). Clonal plaques were picked into $2 \mathrm{~mL}$ of $2 \mathrm{XYT}$ media and expanded overnight, shaking at $230 \mathrm{rpm}$ at $37^{\circ} \mathrm{C}$, filtered, and Sanger sequenced.

tRNA diagrams. R2R was used to generate tRNA diagrams ${ }^{33}$. R2R is free software available from http://www.bioinf.uni-leipzig.de/ zasha/R2R/.

Phage plaque assays
Manual protocol. S2060 cells were transformed with the Accessory Plasmid (AP) of interest. Overnight cultures of single colonies grown in 2XYT media supplemented with maintenance antibiotics were diluted 1,000-fold into fresh 2XYT media with maintenance antibiotics and grown at $37{ }^{\circ} \mathrm{C}$ with shaking at $230 \mathrm{rpm}$ to $\mathrm{OD}_{600} \sim 0.6-0.8$ before use. Bacteriophage were serially diluted 100 -fold (4 dilutions total) in $\mathrm{H}_{2} \mathrm{O} .100$ $\mu \mathrm{L}$ of cells were added to $100 \mu \mathrm{L}$ of each phage dilution, and to this 0.85 $\mathrm{mL}$ of liquid $\left(70^{\circ} \mathrm{C}\right)$ top agar $(2 \mathrm{XYT}$ media $+0.6 \%$ agar $)$ supplemented with $2 \%$ Bluo-Gal (GoldBio, CAS \#97753-82-7) was added and mixed by pipetting up and down once. This mixture was then immediately pipetted onto one quadrant of a quartered petri dish (VWR, 25384308).already containing $2 \mathrm{~mL}$ of solidified bottom agar (2XYT media $+1.5 \%$ agar, no antibiotics). After solidification of the top agar, plates were incubated at $37^{\circ} \mathrm{C}$ for $16-18 \mathrm{~h}$.

Robotics-accelerated protocol. Plaque assays were automated as previously described ${ }^{34}$. Briefly, the same procedure was followed as above, except that plating of the plaque assays was done by a liquid handling robot (Hamilton Robotics) by plating $20 \mu \mathrm{L}$ of bacterial culture and $100 \mu \mathrm{L}$ of phage dilution with $200 \mu \mathrm{L}$ of soft agar onto a well of a 24well plate already containing $235 \mu \mathrm{L}$ of hard agar per well. To prevent premature cooling of soft agar, the soft agar was placed on the deck in a $70^{\circ} \mathrm{C}$ heat block.

Luciferase readthrough assay. Luciferase readthrough assays were performed as previously described ${ }^{36}$. Briefly, S2060 bacteria were transformed with a luciferase reporter containing a quadruplet codon at residue 357 (for example, https://benchling.com/s/ seq-

7CsWcP8Ez4JNjNM9W23N, Addgene \#134787) and an inducible qtRNA expression plasmid (for example, https://benchling.com/s/ seqF5NZDNmxOhUoWp5DX41h, Addgene \#134800). Bacteria were grown overnight, then diluted 500 -fold the next day into DRM media with qtRNA expression induced (1mM IPTG, GoldBio, I2481C5), or suppressed (0mM IPTG). Absorbance and luminescence measurements are taken kinetically in a ClarioSTAR plate reader (BMG Labtech) over the course of $8 \mathrm{~h}$. To account for differential growth rate, all luminescence values are considered at $\mathrm{OD}_{600}=0.5$. The $\%$ of Triplet translation efficiency is calculated using the formula (QuadLuX qtRNA induced - QuadLuX qtRNA uninduced) I (TriLux - QuadLux qtRNA uninduced), where:

- TriLux is the luminescence of the positive control, a luciferase encoded entirely with triplet codons

- QuadLux qtRNA induced is the luminescence produced by the quadruplet codon-bearing reporter upon qtRNA expression (1 mM IPTG)

- QuadLuX qtRNA uninduced is the luminescence produced by the quadruplet codon-bearing reporter in the absence of qtRNA expression (0 mM IPTG)

Growth defect. Our measurement of growth defect involves analysis of the absorbance measured during the $8 \mathrm{~h}$ growth curves taken in the luciferase readthrough assay. We identify the time, $t_{\text {measure }}$ at which QuadLux ${ }_{\text {qtRNA induced reaches }} \mathrm{OD}_{600}=0.5$. Growth defect $=0.5 /(\mathrm{OD}$ of QuadLuX qtRNA suppressed at $t_{\text {measure). }}$.

Phage library primer design. We do not recommend USER cloning for library creation inside of high-secondary structure tRNAs; instead, we used degenerate primers and blunt end ligation. Primers were designed for use with around-the-world PCR, creating a one-piece blunt-end ligation. In order to reduce nucleotide bias during blunt end ligation assembly, the last degenerate base was designed to be at least one base away from the end of the primer. In all cases, phage bearing the wildtype triplet tRNA scaffold are used as a template for the PCR in order to eliminate background bias in the libraries. 
bioRxiv preprint doi: https://doi.org/10.1101/2021.04.26.441066; this version posted April 26, 2021. The copyright holder for this preprint (which was not certified by peer review) is the author/funder, who has granted bioRxiv a license to display the preprint in perpetuity. It is made available under aCC-BY-NC-ND 4.0 International license.

Degenerate NNNN anticodon libraries. Degenerate nucleotides are included at bases $34,35,35.5$ and 36 of the qtRNA. See an example of this primer design: https://benchling.com/s/seq-

$5 \mathrm{k} 2 \mathrm{FZdPbWpiB8tOh8WCK}$

Primer design library diversified at positions 32, 37 and 38 of qtRNA. Degenerate nucleotides are included at bases 32,37 and 38 of the qtRNA. See an example of this primer design:

https://benchling.com/s/seq-oMMGFRI2vITYpNX2HWkz

Phage library cloning. Primers for around-the-world PCR and blunt end ligation were designed as described above. For each library, $200 \mu \mathrm{L}$ of PCR product was produced using Phusion Hotstart Flex polymerase (New England Biolabs, M0535S). The entirety of this PCR product was run on a gel, extracted, and purified using spin column purification (Qiagen, 28106). Background plasmid was digested using Dpn1 (New England Biolabs, R0176L), and the remaining PCR product was purified again using spin columns, and ligated. The ligation product was transformed into competent E. coli S2060 cells containing pJC175e (as described). Transformants were recovered in 2XYT media overnight, shaking at $230 \mathrm{rpm}$ at $37^{\circ} \mathrm{C}$. The phage supernatant from the resulting culture was filtered through a $0.22 \mu \mathrm{m}$ membrane (Thomas Scientific, 1166U41), and plaqued (as described).

plll-based selection of NNNN anticodon libraries. Competent S2060 cells were transformed with a plasmid expressing the appropriate quadruplet codon replacing permissive residue plll-2929. A picked colony was grown overnight in 2XYT media with maintenance antibiotics. Following overnight growth, cultures were diluted 1:1000 in 2XYT media without antibiotics and $500 \mu \mathrm{L}$ of diluted culture was aliquoted into a $2 \mathrm{~mL}$ deep 96-well plate. Wells were inoculated with phage encoding the desired qtRNA library to a final concentration of $10^{5} \mathrm{pfu} / \mathrm{mL}$. The plate was grown overnight, shaking at $230 \mathrm{rpm}$ at $37^{\circ} \mathrm{C}$, and then the phage supernatant was filtered and plaqued in activity-independent host S2060 cells bearing pJC175e. Selections were ranked from lowest final phage titer to highest final phage titer. Two plaques per selection were picked for Sanger sequencing in all selections that enrich over 10-fold, or additional selections as desired.

pIII-based selection and NGS of libraries diversified at positions 32 , 37 and 38. Competent S2060 cells were transformed with a plasmid expressing the appropriate quadruplet codon replacing permissive residue plll-2929. A picked colony was grown overnight in 2XYT media with maintenance antibiotics. Following overnight growth, cultures were diluted 1:1000 in 2XYT media without antibiotics and $500 \mu \mathrm{L}$ of diluted culture was aliquoted into a $2 \mathrm{~mL}$ deep 96 -well plate. Wells were inoculated with phage encoding the desired qtRNA library to a final concentration of $10^{5} \mathrm{pfu} / \mathrm{mL}$. The plate was grown overnight, shaking at $230 \mathrm{rpm}$ at $37^{\circ} \mathrm{C}$, and then the phage supernatant was filtered and plaqued in activity-independent host $\mathrm{S} 2060$ cells bearing pJC175e. Libraries were amplified using PCR and sequenced on a MiSeq to $>10 x$ library size.
Quantification of qtRNA charging using mass spectrometry. Each qtRNA (for example, https://benchling.com/s/seqBpqbupgoHvZu0gFmNUBm) was co-expressed with C-terminal 6xHistagged sfGFP (for example, https://benchling.com/s/seq-

bl1bixktGKegGwboMYIP) with the appropriate quadruplet codon replacing permissive residue 151 in S2060 cells. GFP was purified from these cultures at either $5 \mathrm{~mL}$ or $1 \mathrm{~L}$ scale (Table S2).

Purification at $4 \mathrm{~mL}$ scale. Co-transform bacteria with sfGFP-151-quad and qtRNA expression plasmids. Inoculate a single colony of the expression strain in DRM media (with $100 \mathrm{ug} / \mathrm{ml}$ spectinomycin, $30 \mathrm{ug} / \mathrm{ml}$ kanamycin, and $25 \mathrm{mM}$ glucose) as the seed culture; grow overnight at $37^{\circ} \mathrm{C}, 200 \mathrm{rpm}$. Dilute 1:1000 and into DRM media that induces qtRNA expression (with $100 \mathrm{ug} / \mathrm{ml}$ spectinomycin, $30 \mathrm{ug} / \mathrm{ml}$ kanamycin, and 1 mM IPTG) and grow for 29 hours, 200rpm, at 37C. Cultures were spun down at $5000 \mathrm{~g}$ for 10 minutes and the pellet frozen at $-80 \mathrm{C}$. Thawed pellets were lysed using B-PER Complete Reagent (Thermofisher 89821) and His-tagged protein was purified from cell lysate using a Ni-NTA spin column (Qiagen, 31014). The resulting product was run on a $12 \%$ BisTris PAGE gel (Thermofisher, NP0342PK2), and the appropriate band was extracted for mass spec analysis.

Purification at $1 \mathrm{~L}$ scale. Proteins were purified by BiologicsCorp at $1 \mathrm{~L}$ scale. Inoculate a single colony of the expression strain in LB media (with $100 \mathrm{ug} / \mathrm{ml}$ spectinomycin, $30 \mathrm{ug} / \mathrm{ml}$ kanamycin, and $25 \mathrm{mM}$ glucose) as the seed culture; at $37^{\circ} \mathrm{C}, 200 \mathrm{rpm}$, overnight. Perform $1 \%$ inoculation into $500 \mathrm{ml}$ TB media (with $100 \mathrm{ug} / \mathrm{ml}$ spectinomycin, 30ug/ml kanamycin, and $25 \mathrm{mM}$ glucose); at $37^{\circ} \mathrm{C}, 200 \mathrm{rpm}$, till $\mathrm{OD}_{600}=0.6-0.8$. Add IPTG to a final concentration of $1 \mathrm{mM}$. Continue culturing for $4 \mathrm{hrs}$. Collect cells by centrifugation $\left(4^{\circ} \mathrm{C}, 8,000 \mathrm{rpm}, 15 \mathrm{~min}\right)$. Wash cells once by resuspension with PBS buffer and again collect cells by centrifugation. Resuspend per gram of wet cell pellet with $20 \mathrm{ml}$ Lysis Buffer T300-20L (50 mM Tris- $\mathrm{HCl} \mathrm{pH} \mathrm{8.0,300} \mathrm{mM} \mathrm{NaCl,} \mathrm{and} 20 \mathrm{mM}$ imidazol, plus $1 \mathrm{mM}$ DTT, $1 \%$ Triton X-114, $1 \mathrm{ug} / \mathrm{ml}$ Pepstatin A, and $1 \mathrm{ug} / \mathrm{ml}$ Leupeptin). Sonicate the cell suspension on ice for $15-25$ min ( $500 \mathrm{~W}$; $3 \mathrm{sec}$ burst and $6 \mathrm{sec}$ pause). Centrifuge the cell lysate; at $4 \mathrm{C}, 12500 \mathrm{rpm}$, for 15 minutes. Filter the supernatant through a 0.45 um pore membrane. Purify His-tagged protein using a Ni-IDA column. Analyze fractions by SDSPAGE. For samples with satisfactory purity, pool fractions and dialyzed against PBS buffer ( $\mathrm{pH} \mathrm{7.4);} \mathrm{at} \mathrm{4C;} 2$ hr per cycle, 3-4 cycles. Filter the protein solution through a 0.22 um pore membrane. Concentrate if necessary.

Mass spectrometry analysis. Samples were trypsin digested and measured using HPLC-MS/MS. To analyze the results, the resulting fragmentation spectra were correlated against a custom database containing the predicted fragmentation pattern for the fragment straddling residues 151 if it contained each of the 20 canonical amino acids. Abundance of each species was quantified by calculating the area under the curve of the ion chromatogram for each peptide precursor. The limit of detection was $10^{4}[\mathrm{AU}]$, the lower limit for area under the curve for a peptide on this instrument. 
bioRxiv preprint doi: https://doi.org/10.1101/2021.04.26.441066; this version posted April 26, 2021. The copyright holder for this preprint (which was not certified by peer review) is the author/funder, who has granted bioRxiv a license to display the preprint in perpetuity. It is made available under aCC-BY-NC-ND 4.0 International license.

Figure S1 - Codon reassignment for asparagine and methionine

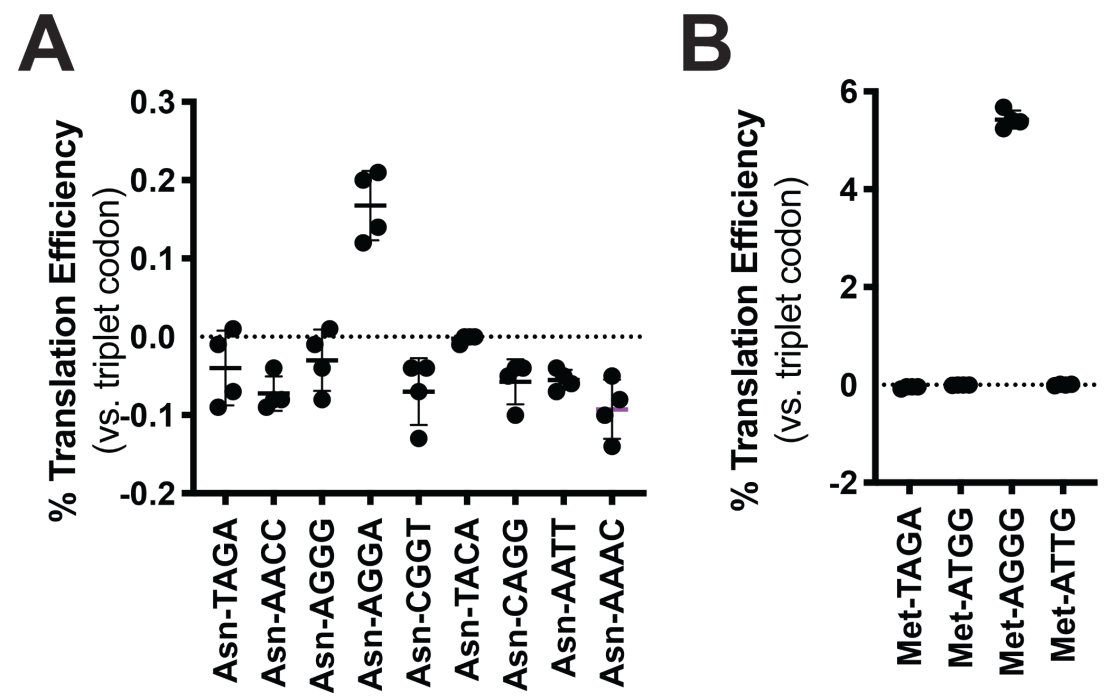

Quadruplet codon experimentation for asparagine and methionine. Measurements are taken kinetically and normalized to culture density. Efficiency is reported relative to luminescence produced by a WT, all triplet luciferase transcript. A) Characterization of nine unique quadruplet codons within the tRNA ${ }^{\text {Asn }}$ scaffold. B) Characterization of four quadruplet codons within the tRNA ${ }^{\text {Met }}$ scaffold. 
bioRxiv preprint doi: https://doi.org/10.1101/2021.04.26.441066; this version posted April 26, 2021. The copyright holder for this preprint (which was not certified by peer review) is the author/funder, who has granted bioRxiv a license to display the preprint in perpetuity. It is made available under aCC-BY-NC-ND 4.0 International license.

\section{Table S1 - Table of tRNA scaffolds}

\begin{tabular}{|c|c|c|}
\hline tRNA & $\begin{array}{l}\text { Sequence }(\text { bold caps = anticodon; } \\
\text { bold = positions } 32,37,38 \text { ) }\end{array}$ & $\begin{array}{l}\text { Expression plasmid map } \\
\text { (IPTG inducible in S2060 strain) }\end{array}$ \\
\hline tRNA $A^{\text {Ala }}$ & $\begin{array}{l}\text { ggggctatagctcagctgggagagcgcttgcatGGCatgcaagag } \\
\text { gtcagcggttcgatcccgcttagctccacca }\end{array}$ & https://benchling.com/s/seq-tg2Ok8ejpnqNNR1RCgxJ \\
\hline tRNA ${ }^{\text {Arg }}$ & $\begin{array}{l}\text { gcatccgtagttcagctggatagagtactcggctACGaaccgagcg } \\
\text { gtcggaggttcgaatcctcccggatgcacca }\end{array}$ & https://benchling.com/s/seq-5Z1JRvAM5CUKFL2CyQHI \\
\hline tRNA ${ }^{\text {Asn }}$ & $\begin{array}{l}\text { tcctctgtagttcagtcggtagaacggcggactGTTaatccgtatgtc } \\
\text { actggttcgagtccagtcagaggagcca }\end{array}$ & https://benchling.com/s/seq-cvpe4WFNdq3urdBOErUE \\
\hline tRNA ${ }^{\text {Asp }}$ & $\begin{array}{l}\text { ggagcggtagttcagtcggttagaatacctgcctGTCacgcagggg } \\
\text { gtcgcgggttcgagtcccgtccgttccgcca }\end{array}$ & https://benchling.com/s/seq-cvcp7xMH4VVbQxCWY57a \\
\hline tRNA ${ }^{\text {Cys }}$ & $\begin{array}{l}\text { ggcgcgttaacaaagcggttatgtagcggattGCAaatccgtctagt } \\
\text { ccggttcgactccggaacgcgcctcca }\end{array}$ & https://benchling.com/s/seq-pCOWJ5Ba9vyLDMfhXXTr \\
\hline tRNA ${ }^{\text {Glu }}$ & $\begin{array}{l}\text { gtccccttcgtctagaggcccaggacaccgccctTTCacggcggta } \\
\text { acaggggttcgaatcccctaggggacgcca }\end{array}$ & https://benchling.com/s/seq-cYNon2BOYXiyMus5Egj2 \\
\hline tRNA $A^{G \ln }$ & $\begin{array}{l}\text { tggggtatcgccaagcggtaaggcaccggattCTGattccggcattc } \\
\text { cgaggttcgaatcctcgtaccccagcca }\end{array}$ & https://benchling.com/s/seq-1iWjqPpZE7TQZ4cWEbKI \\
\hline tRNA ${ }^{\text {Gly }}$ & $\begin{array}{l}\text { gcgggcgtagttcaatggtagaacgagagcttCCCaagctctatac } \\
\text { gagggttcgattcccttcgeccgctcca }\end{array}$ & https://benchling.com/s/seq-XFAUpIPfQA8J3y3eKe9o \\
\hline tRNA ${ }^{\text {His }}$ & $\begin{array}{l}\text { gtggctatagctcagttggtagagccctggattGTGattccagttgtcg } \\
\text { tgggttcgaatcccattagcсaсcсca }\end{array}$ & https://benchling.com/s/seq-xS0QHSq6wlaNBGG12Vxl \\
\hline tRNA ${ }^{\text {lle }}$ & $\begin{array}{l}\text { aggcttgtagctcaggtggttagagcgcaccctGATaagggtgag } \\
\text { gtcggtggttcaagtccactcaggcctacca }\end{array}$ & https://benchling.com/s/seq-cUTbLsat2L7hQcjOZxXW \\
\hline tRNA ${ }^{\text {Leu }}$ & $\begin{array}{l}\text { gccgaagtggcgaaatcggtagacgcagttgattCAAaatcaacc } \\
\text { gtagaaatacgtgccggttcgagtccggccttcggcacca }\end{array}$ & https://benchling.com/s/seq-PSGYeWnss5uNAMjGNWk9 \\
\hline tRNA ${ }^{\text {Lys }}$ & $\begin{array}{l}\text { gggtcgttagctcagttggtagagcagttgactTTTaatcaattggtcg } \\
\text { caggttcgaatcctgcacgacccacca }\end{array}$ & https://benchling.com/s/seq-vsDR689MSNPR3k4lsy8I \\
\hline tRNA $^{\text {Met }}$ & $\begin{array}{l}\text { ggctacgtagctcagttggttagagcacatcactCATaatgatggggt } \\
\text { cacaggttcgaatcccgtcgtagccacca }\end{array}$ & $\underline{\text { https://benchling.com/s/seq-NUddULsETeJ7xYfqySrf }}$ \\
\hline tRNA ${ }^{\text {fMet }}$ & $\begin{array}{l}\text { cgcggggtggagcagcctggtagctcgtcgggctCATaacccgaa } \\
\text { ggtcgtcggttcaaatccggeccccgcaacca }\end{array}$ & https://benchling.com/s/seq-kKD5TUaRnzoDXYhJBfdf \\
\hline $\mathrm{tRNA}^{\mathrm{Phe}}$ & $\begin{array}{l}\text { gcccggatagctcagtcggtagagcaggggattGAAaatccccgtg } \\
\text { tccttggttcgattccgagtccgggcacca }\end{array}$ & https://benchling.com/s/seq-W61lkhltEYkNdtNOTG5h \\
\hline tRNA $A^{\text {Pro }}$ & $\begin{array}{l}\text { cggtgattggcgcagcctggtagcgcacttcgttCGGgacgaaggg } \\
\text { gtcggaggttcgaatcctctatcaccgacca }\end{array}$ & https://benchling.com/s/seq-2vdHeJ6LQJyDgaX3uiZv \\
\hline tRNA ${ }^{\text {Ser }}$ & $\begin{array}{l}\text { ggagagatgccggagcggctgaacggaccggtctCGAaaaccg } \\
\text { gagtaggggcaactctaccgggggttcaaatccccctctctccgcca }\end{array}$ & https://benchling.com/s/seq-cGi0J8BtBUydV1m1uq2S \\
\hline $\mathrm{tRNA}^{\mathrm{Thr}}$ & $\begin{array}{l}\text { gccgatatagctcagttggtagagcagcgcattCGTaatgcgaagg } \\
\text { tcgtaggttcgactcctattatcggcacca }\end{array}$ & https://benchling.com/s/seq-QR5vD3dJn5S23lbSXpMp \\
\hline tRNA $A^{T r p}$ & $\begin{array}{l}\text { aggggcgtagttcaattggtagagcaccggtctCCAaaaccgggtg } \\
\text { ttgggagttcgagtctctccgeccctgcca }\end{array}$ & https://benchling.com/s/seq-PsQbqBCffQ1CVLU6tdIK \\
\hline tRNA ${ }^{\text {Tyr }}$ & $\begin{array}{l}\text { ggtggggttcccgagcggccaaagggagcagactGTAaatctgcc } \\
\text { gtcacagacttcgaaggttcgaatccttcccccaccacca }\end{array}$ & https://benchling.com/s/seq-8fpqOoBdg536ImikNeYy \\
\hline tRNA $A^{\text {Val }}$ & $\begin{array}{l}\text { gcgtccgtagctcagttggttagagcaccaccttGACatggtggggg } \\
\text { tcggtggttcgagtccactcggacgcacca }\end{array}$ & https://benchling.com/s/seq-LROPe63eAegmXN9pKMWp \\
\hline
\end{tabular}

Canonical triplet tRNAs were cloned from E. coli K12 genomic DNA. Body text Figures 1 and 2 concern mutations to the anticodon (bolded caps); body text Figure 3 concerns mutation to positions 32, 37 and 38 (bolded lower case). We use qtRNA ${ }^{\text {three letter scaffold }}$ four letter DNA codon nomenclature to refer to qtRNAs; e.g. to create qtRNA ${ }^{\text {Ser }}{ }_{\text {TAGA, }}$, the CGA anticodon in TRNA ${ }^{\text {Ser }}$ scaffold would be replaced by TCTA. 
bioRxiv preprint doi: https://doi.org/10.1101/2021.04.26.441066; this version posted April 26, 2021. The copyright holder for this preprint (which was not certified by peer review) is the author/funder, who has granted bioRxiv a license to display the preprint in perpetuity. It is made available under aCC-BY-NC-ND 4.0 International license.

Table S2 - purification scale of qtRNA-translated proteins

\begin{tabular}{|c|c|c|c|c|c|}
\hline qtRNA & $\begin{array}{l}\text { Purification } \\
\text { scale }\end{array}$ & Result & $\begin{array}{l}\text { Amino Acid } \\
\text { Incorporation }\end{array}$ & $\begin{array}{l}\text { Limit of } \\
\text { detection }\end{array}$ & $\begin{array}{l}\text { Expression plasmid map } \\
\text { (IPTG inducible in S2060 E. coli strain) }\end{array}$ \\
\hline Ala-AGGG-32T-38G & $1 \mathrm{~L}$ & Purified and measured sample & $\begin{array}{l}77 \% \text { Arg; } \\
22 \% \text { Ala }\end{array}$ & $0.002 \%$ & https://benchling.com/s/seq-MDPm0wgpoZcbQbgcrecA \\
\hline Asn-AGGA & $1 \mathrm{~L}$ & Yield too low to measure & - & - & https://benchling.com/s/seq-xWG68x7wR1CshAE1tV9| \\
\hline Asp-CGGC-32G-38A & $1 \mathrm{~L}$ & Purified and measured sample & $100 \% \operatorname{Arg}$ & $0.002 \%$ & https://benchling.com/s/seq-7thPCofT0Jhr9UB7h4OL \\
\hline Cys-CGGC & $1 \mathrm{~L}$ & Yield too low to measure & - & - & https://benchling.com/s/seq-rHgdjGw6XdyEYp84L6J7 \\
\hline GIn-CAGG & $5 \mathrm{~mL}$ & Purified and measured sample & $100 \% \mathrm{Gln}$ & $0.002 \%$ & https://benchling.com/s/seq-tGyQojadJ2mRCj411540 \\
\hline Glu-CGGT & $5 \mathrm{~mL}$ & Purified and measured sample & $100 \%$ Glu & $0.1 \%$ & https://benchling.com/s/seq-nr3CInJvm9MWjnGcvy4s \\
\hline Gly-GGGG & $5 \mathrm{~mL}$ & Purified and measured sample & $100 \%$ Gly & $0.0003 \%$ & https://benchling.com/s/seq-Ry0fYxluloLOYvXj8XxQ \\
\hline His-TACA-32G & $1 \mathrm{~L}$ & $\begin{array}{l}\text { Purified at } 1 \mathrm{~L} \text { scale, but no } \\
\text { peptide detected in mass spec }\end{array}$ & - & - & https://benchling.com/s/seq-HOf86rVgpat27IDVvCxY \\
\hline Ile-AGGA & $1 \mathrm{~L}$ & Purified and measured sample & $100 \% \operatorname{Arg}$ & $0.005 \%$ & https://benchling.com/s/seq-hbgRy8yUWsOtbdbpmdP1 \\
\hline Leu-AGGG & $1 \mathrm{~L}$ & Yield too low to measure & - & - & https://benchling.com/s/seq-V8jP2xPq1V9v10Ps3cHI \\
\hline Lys-AGAA & $1 \mathrm{~L}$ & Yield too low to measure & - & - & https://benchling.com/s/seq-fyjM4W7dLZI6RBOHKzwr \\
\hline Met-AGGG & $1 \mathrm{~L}$ & Purified and measured sample & $\begin{array}{c}96 \% \text { Arg; } \\
3 \% \text { Met; } \\
2 \% \text { Gly }\end{array}$ & $0.002 \%$ & https://benchling.com/s/seq-T9ehlv5N1lolyU4sX9V7 \\
\hline Phe-CGGC & $5 \mathrm{~mL}$ & Purified and measured sample & $100 \%$ Phe & $0.8 \%$ & https://benchling.com/s/seq-fgpEdowxfsOdeuYLgs5V \\
\hline Pro-CCGG & $1 \mathrm{~L}$ & Purified and measured sample & $100 \%$ Pro & $0.009 \%$ & https://benchling.com/s/seq-T1vdy3JSiZ2DNG91TwA6 \\
\hline Ser-TCGG-32A-38C & $1 \mathrm{~L}$ & Purified and measured sample & $100 \%$ Ser & $0.04 \%$ & https://benchling.com/s/seq-TWCRTU1TcjS0u19SnKEW \\
\hline Thr-ACGG & $1 \mathrm{~L}$ & Yield too low to measure & - & - & https://benchling.com/s/seq-cJDL7NuzsBDMZnkLzEpL \\
\hline Trp-AGGG & $1 \mathrm{~L}$ & Purified and measured sample & $100 \% \operatorname{Arg}$ & $0.2 \%$ & https://benchling.com/s/seq-g8megLDGGL2LIvGVyJFi \\
\hline Val-CGGC-32T-38A & $1 \mathrm{~L}$ & Purified and measured sample & $100 \% \operatorname{Arg}$ & $0.005 \%$ & https://benchling.com/s/seq-i3tcy29Qtmv8ru3xLvb5 \\
\hline $\begin{array}{l}\text { Tyr-, Arg-, and other } \\
\text { TAGA qtRNAs }{ }^{36}\end{array}$ & $5 \mathrm{~mL}$ & Purified and measured sample & Assorted & Assorted & \\
\hline
\end{tabular}

For sfGFP purification, qtRNA expression plasmids (listed above) were co-expressed with C-terminal 6xHis-tagged sfGFP with the appropriate quadruplet codon replacing permissive residue 151. For example, sfGFP-151-GGGG, https://benchling.com/s/seq-b/1bixktGKegGwboMYIP 